\section{Revista de CIENCIAS AMBIENTALES Tropical Journal of Environmental Sciences}

Revista de Ciencias Ambientales (Trop J Environ Sci). EISSN: 2215-3896.

(Enero-Junio, 2019) . Vol 53(1): 1-22.

Dol: http://dx.doi.org/10.15359/rca.53-1.1

Open Access: www.revistas.una.ac.cr/ambientales

EMAIL: revista.ambientales@una.ac.cr

Lafuente W., Soto L. M., López C., Domínguez-Granda L.

\title{
Efectos de un derrame de petróleo crudo en la comunidad de macroinvertebrados bentónicos de un río amazónico ecuatoriano
}

\author{
Effects of a Crude Oil Spill in the Benthic Macroinvertebrate Community of an \\ Ecuadorian Amazon River
}

Wilson Lafuente ${ }^{a}$, Luz Marina Soto ${ }^{b}$, Carlos López $^{c}$, Luis Domínguez-Granda ${ }^{d}$

[Recibido: 8 de agosto 2018, Aceptado: 20 de octubre 2018, Corregido: 11 de noviembre 2018, Publicado: 1 de enero 2019]

\begin{abstract}
Resumen
Los derrames de petróleo constituyen una fuente importante de perturbación de los macroinvertebrados bentónicos de aguas continentales. Sin embargo, los estudios referentes a estas perturbaciones en las regiones tropicales, y particularmente en el Neotrópico, son limitados. En el presente trabajo se evaluaron los efectos de un derrame de 11700 barriles de petróleo en las comunidades de macroinvertebrados bentónicos de los ríos Santa Rosa, Quijos y Coca, amazonia ecuatoriana, mediante el uso de índices bióticos, variables de calidad de agua y sedimentos, tanto en sitios impactados como en sitios de referencia en los años 2011 y 2012. Las correlaciones de Spearman establecieron relaciones negativas significativas $(\mathrm{p}<0.05)$ entre la variable "hidrocarburos totales de petróleo" y los taxa Ephemeroptera-Plecoptera-Trichoptera (índice EPT), Trichoptera, y la familia Elmidae. Se estableció una relación inversa entre el índice SIGNAL2 con los hidrocarburos totales de petróleo. El procedimiento de permutación de respuesta múltiple determinó una diferencia entre las comunidades de macroinvertebrados de estaciones de referencia e impactadas del año 2011, y entre las comunidades de macroinvertebrados de estaciones de referencia año 2011 y estaciones impactadas año 2012. El escalamiento multidimensional no-métrico implicó usar tres dimensiones, en el cual el eje dos estuvo mejor relacionado con los hidrocarburos totales de petróleo $(r=0.45)$. Estos resultados sugieren que factores distintos a hidrocarburos totales de petróleo son los responsables de la composición heterogénea de las comunidades de macroinvertebrados.
\end{abstract}

Palabras clave: Índices bióticos; Neotrópico; recuperación ecológica.

\section{Abstract}

Oil spills are a major source of disturbance to benthic macroinvertebrates from inland waters. However, studies regarding these disturbances in tropical regions, and particularly in the Neotropics, are limited. This study evaluates the effects of a 11 700-barrel heavy crude oil spill on the benthic macroinvertebrate community of Santa Rosa,

a Especialista en ciencias ambientales; académico de la Facultad de Ciencias Naturales y de la Agricultura, Universidad Estatal del Sur de Manabí (UNESUM), Ecuador, wilson.lafuente@unesum.edu.ec

b Especialista en microbiología de aguas continentales; investigadora y académica del Departamento de Biología, Facultad Experimental de Ciencias, Universidad del Zulia, Ecuador, lusoto@espol.edu.ec

c Especialista en ecología de aguas continentales; investigador del Centro del Agua y Desarrollo Sustentable, Escuela Superior Politécnica del Litoral (ESPOL), Ecuador, cllopez@espol.edu.ec

d Especialista en manejo ambiental; investigador y académico de la Facultad de Ciencias Naturales y Matemáticas, Escuela Superior Politécnica del Litoral (ESPOL), Ecuador, ldomingu@espol.edu.ec

(c)

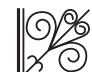

euna 


\section{Revista de CIENCIAS AMBIENTALES Tropical Journal of Environmental Sciences}

Revista de Ciencias Ambientales (Trop J Environ Sci). EISSN: 2215-3896. (Enero-Junio, 2019) . Vol 53(1): 1-22. D0l: http://dx.doi.org/10.15359/rca.53-1.1 Open Access: www.revistas.una.ac.cr/ambientales EMAIL: revista.ambientales@una.ac.cr Lafuente W., Soto L. M., López C., Domínguez-Granda L.

Quijos and Coca rivers, Ecuadorian Amazon. The evaluation used a biotic index and water quality and sediments variables, both in the impacted sites and in the reference sites during two field trips in 2011 and 2012. Spearman correlations established significant negative relationships $(\mathrm{p}<0.05)$ between total petroleum hydrocarbons and Ephemeroptera-Plecoptera-Trichoptera (EPT index), Trichoptera, and Elmidae family taxa. An inverse relationship was established between the SIGNAL2 index and total petroleum hydrocarbons. A multiple response permutation procedure (MRPP) determined a difference between the macroinvertebrate communities of reference and the impacted stations in 2011, and between the macroinvertebrate communities of reference stations 2011 and stations impacted in 2012. Non-metric multidimensional scaling involved using three dimensions, in which axis two was better related to total petroleum hydrocarbons $(\mathrm{r}=0.45)$. These results suggest that factors other than total petroleum hydrocarbons are responsible for the heterogeneous composition of the macroinvertebrate communities.

Keywords: biotic indices; ecological recovery; Neotropics.

\section{Introducción}

Comparada con la extensa bibliografía sobre derrames de petróleo en el mar, las investigaciones sobre los efectos de estos sucesos en ecosistemas de agua dulce son limitadas, la mayoría realizadas en los Estados Unidos. Estas han descrito una amplia variedad de respuestas en los macroinvertebrados de ríos, tales como pérdida de comunidades bentónicas de un río de Texas, 26 meses después de un derrame de 254401 de crudo (Harrel, 1985); ausencia de algunas especies de Ephemeroptera, Plecoptera y Trichoptera en el arroyo Asher, nueve meses después de un derrame de 1.5 millones de litros de petróleo crudo doméstico (Crunkilton y Duchrow, 1990); y baja diversidad de macroinvertebrados de hábitats de remanso del río Gasconade, 18 meses después de un derrame de 3.3 millones de litros de petróleo (Poulton et al., 1997).

En adición a los estudios anteriormente mencionados, es importante destacar la contribución de algunos trabajos que han evaluado los impactos de derrames de petróleo sobre los macroinvertebrados en condiciones de laboratorio, incluidos: reducción de sobrevivencia del Ephemeroptera Hexagenia bilineata expuesto a sedimentos con residuos de hidrocarburos (Ort et al., 1995); disminución significativa en la abundancia de nueve taxones de quironómidos a determinadas concentraciones de hidrocarburos totales de petróleo en sedimentos (Pettigrove y Hoffmann, 2005); y retraso en la emergencia de Chironomus riparius durante la exposición crónica a sedimentos con compuestos aromáticos policíclicos (Paumen et al., 2008).

Los estudios de los efectos de derrames de petróleo en los macroinvertebrados de ríos neotropicales son aún más escasos. En América del Sur, solo se ha publicado un estudio que informó una reducción en la abundancia y riqueza de invertebrados edáficos del banco del río Cururu, Brasil, afectado por un derrame de petróleo (Couceiro et al., 2007). Para el caso de Ecuador, los pocos estudios corresponden a los efectos del derrame de petróleo en comunidades planctónicas de ecosistemas lacustres (Cueva, 2013; Molina, 2013). Solo recientemente se evaluó la habilidad de los insectos acuáticos de ríos de páramo ecuatoriano para evitar diferentes concentraciones de hidrocarburos aromáticos policíclicos, por medio del transporte de las corrientes de agua. Para el Trichoptera Anomalocosmoecus palugillensis, el movimiento de las corrientes de

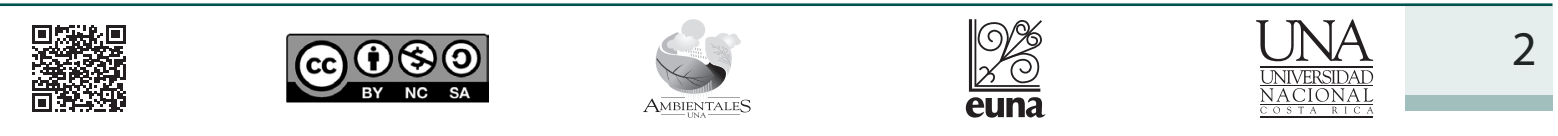




\section{Revista de CIENCIAS AMBIENTALES Tropical Journal of Environmental Sciences}

Revista de Ciencias Ambientales (Trop J Environ Sci). EISSN: 2215-3896. (Enero-Junio, 2019) . Vol 53(1): 1-22. Dol: http://dx.doi.org/10.15359/rca.53-1.1 Open Access: www.revistas.una.ac.cr/ambientales EMAlL: revista.ambientales@una.ac.cr Lafuente W., Soto L. M., López C., Domínguez-Granda L.

agua fue la mayor causa de declinación poblacional en el ensayo con baja concentración de este tipo de hidrocarburo (Araújo, 2014). La escasez de estudios realizados y la utilización de índices desarrollados en zonas templadas para evaluar las respuestas de los macroinvertebrados ante derrames de petróleo en el Neotrópico hacen necesaria la realización de estudios que permitan probar estos índices o desarrollar índices propios para la región.

El 25 de febrero de 2009, un movimiento de tierra produjo la rotura de un tramo del Oleoducto de Crudos Pesados (OCP), lo que provocó el derrame de 11700 barriles de crudo en el río Santa Rosa, provincia de Napo. Debido a las condiciones climáticas de ese momento, el crudo se desplazó $185 \mathrm{~km}$ aguas abajo hacia los ríos Quijos y Coca. Para el 12 de marzo de ese mismo año, OCP Ecuador S.A. superó la emergencia y comenzó las labores de limpieza y remediación ambiental, y subsecuente monitoreo interno seguido por personal del Ministerio del Ambiente del Ecuador (MAE), se culminó el 30 de septiembre de 2009 (Alemán et al., 2010; Mendizábal et al., 2012).

El objetivo principal de este estudio consistió en evaluar, mediante el uso de índices bióticos basados en comunidades de macroinvertebrados bentónicos, el nivel de recuperación ecológica de los ríos Santa Rosa, Quijos y Coca que han sido afectados por el derrame de hidrocarburos.

\section{Materiales y métodos}

\subsection{Sitio de estudio}

El río Santa Rosa se encuentra localizado en la provincia de Napo, Ecuador $\left(0^{\circ} 13^{\prime} 43^{\prime \prime} \mathrm{S}\right.$,

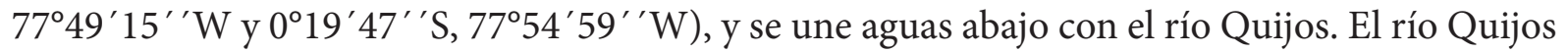
se une aguas abajo con el río Salado para formar el río Coca. El derrame de petróleo se originó en el río Santa Rosa y se extendió aguas abajo hacia los ríos Quijos y Coca. Las estaciones de muestreo fueron ubicadas con ayuda de cartas topográficas del área (escala 1:50 000) y un sistema de posicionamiento global portátil Garmin GPSMAP ${ }^{\circledR} 64$ y cumplieron los siguientes criterios: 1) fácil acceso, 2) las estaciones de referencia del río Santa Rosa (códigos E1 y E2) se ubicaron aguas arriba del sitio del derrame de petróleo, 3) las estaciones de referencia del río Quijos (códigos E6, E7 y E8) se ubicaron aguas arriba de la unión con el río Santa Rosa, lo suficientemente alejadas de la influencia de las poblaciones, 4) las estaciones impactadas se ubicaron aguas abajo del sitio del derrame de petróleo, en los ríos Santa Rosa (códigos E3, E4 y E5) y Coca (códigos E9, E10 y E11). En la Figura 1 se indica la ubicación de las estaciones de muestreo en cada uno de los ríos estudiados. La estación de muestreo E3 se ubicó cerca al sitio donde ocurrió el derrame de petróleo. Los sitios E6, E7, E8, E1 y E2 estuvieron a 29, 27, 25, 2,5 y $1 \mathrm{~km}$ sobre el sitio donde se originó el derrame, y los sitios E4, E5, E9, E10 y E11 estuvieron a uno, dos, 32, 33 y $39 \mathrm{~km}$ bajo el sitio de derrame.

Según la escala de orden de río (Strahler, 1957), en los puntos de muestreo, el río Santa Rosa es de primer y segundo orden, el río Quijos es de cuarto orden, y el río Coca es de quinto orden.

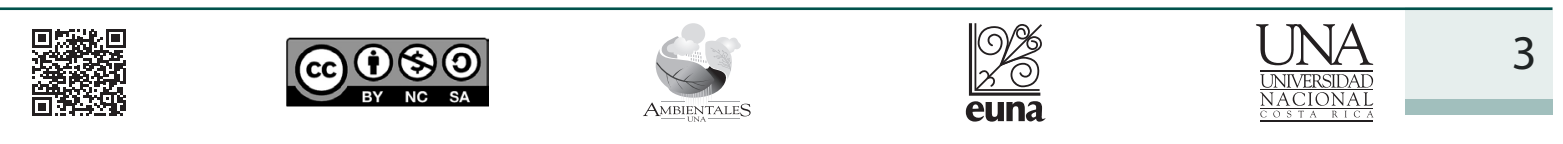




\section{Revista de} CIENCIAS AMBIENTALES Tropical Journal of Environmental Sciences
Revista de Ciencias Ambientales (Trop J Environ Sci). EISSN: 2215-3896.

(Enero-Junio, 2019) . Vol 53(1): 1-22.

D0l: http://dx.doi.org/10.15359/rca.53-1.1

Open Access: www.revistas.una.ac.cr/ambientales

EMAIL: revista.ambientales@una.ac.cr Lafuente W., Soto L. M., López C., Domínguez-Granda L.

Estas estaciones de muestreo no están catalogadas en los planes de monitoreo de la cuenca que efectuó el MAE posterior a las labores de remediación de la zona afectada.

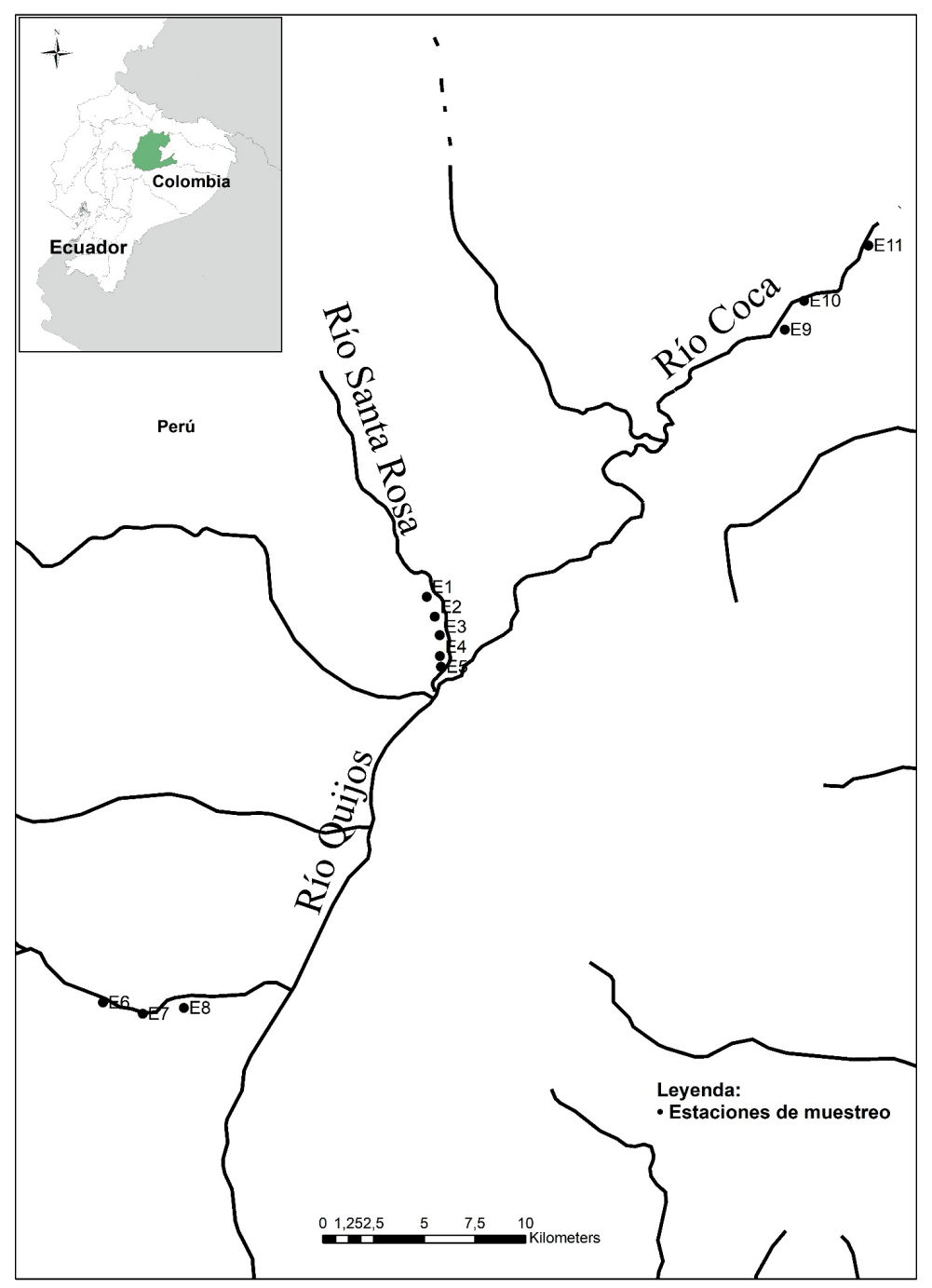

Figura 1. Estaciones de muestreo de los ríos Quijos, Santa Rosa y Coca, ubicados en la provincia de Napo, Ecuador.

\subsection{Métodos de campo}

Se efectuaron dos salidas de campo en los meses de septiembre de 2011 y enero de 2012, después de 31 meses del derrame de petróleo. Se midió in situ temperatura, oxígeno disuelto (OD), saturación de OD, y conductividad, mediante el uso de una sonda multiparámetro YSI modelo 85; y potencial de hidrógeno, con una sonda medidor YSI modelo ph10. Para determinar

\begin{tabular}{|c|c|}
\hline 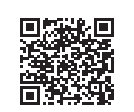 & (c) (i) (5) (O) \\
\hline
\end{tabular}




\section{Revista de CIENCIAS AMBIENTALES Tropical Journal of Environmental Sciences}

Revista de Ciencias Ambientales (Trop J Environ Sci). EISSN: 2215-3896. (Enero-Junio, 2019) . Vol 53(1): 1-22. Dol: http://dx.doi.org/10.15359/rca.53-1.1 Open Access: www.revistas.una.ac.cr/ambientales EMAlL: revista.ambientales@una.ac.cr Lafuente W., Soto L. M., López C., Domínguez-Granda L.

fosfato $\left(\mathrm{PO}_{4}^{3-}\right)$ se utilizó kits colorimétricos Aquaquant ${ }^{\oplus}$. Las muestras de macroinvertebrados bentónicos fueron colectadas en tramos de río de 20 a $30 \mathrm{~m}$ de longitud con ayuda de una red cónica $(20 \times 30 \mathrm{~cm}, 400 \mu \mathrm{m})$ instalada en un marco de hierro en forma de $\mathrm{D}(30 \mathrm{x} 30 \mathrm{~cm})$, para lo cual se disturbó el substrato del río con el pie en frente de la red abierta contra la corriente por un lapso de tres minutos, tanto en aguas rápidas como en aguas lentas poco profundas $(<0.5$ m) (De Pauw y Vanhooren, 1983). Las muestras fueron almacenadas en recipientes de plástico y preservadas en alcohol al $70 \%$ para ser analizadas posteriormente en laboratorio.

\subsection{Métodos de laboratorio}

Las muestras de agua fueron recolectadas de cada sitio de muestreo con botellas ámbar de $500 \mathrm{ml}$, y seguidamente se las almacenó en una hielera. Se midió ex situ nitrato $\left(\mathrm{NO}_{3}^{-}\right)$, nitrito $\left(\mathrm{NO}_{2}^{-}\right)$y alcalinidad $\left(\mathrm{CaCO}_{3}\right)$, mediante el uso de un fotómetro YSI modelo 9 100. Se midió demanda química de oxígeno (DQO) por medio del método colorimétrico de reflujo cerrado, y sólidos suspendidos totales por medio de secado a $103-105^{\circ} \mathrm{C}$ (A.P.H.A., 2005). Las muestras de sedimento fueron recolectadas en aguas lentas poco profundas $(<0.5 \mathrm{~m})$ con ayuda de una pala metálica y, posteriormente, almacenadas en fundas Ziploc (modificado de Poulton et al., 1997). Se midieron niveles de hidrocarburos totales de petróleo (HTP) en muestras de sedimento mediante el método EPA 418.2.

Las muestras preservadas de macroinvertebrados bentónicos fueron tamizadas con diferente ojo de malla (2 000, 1 000, 750, 500 y $350 \mu \mathrm{m})$ (De Pauw y Vanhooren, 1983). La fracción retenida en cada tamiz fue gradualmente transferida a una bandeja blanca metálica (30 x 50 $\mathrm{cm}$ ), con el fin de separar los macroinvertebrados y, posteriormente, identificarlos a nivel de familia con ayuda de un estereomicroscopio a una magnificación de 10 y claves de identificación taxonómica (Domínguez y Fernández, 2009; Roldán, 1988).

\subsection{Análisis de datos}

Medidas de abundancia, diversidad, riqueza de taxa, medidas de composición, e índices bióticos, fueron aplicados a las comunidades de macroinvertebrados bentónicos de los ríos Santa Rosa, Quijos y Coca. Se utilizó el análisis de correlación de Spearman para determinar correlaciones estadísticas en índices bióticos, variables ambientales, y comunidades de macroinvertebrados. Este análisis fue ejecutado con el software STATISTICA 7, considerando $\mathrm{p}<0.05$ (Zar, 1996).

Se utilizó el análisis de agrupación procedimiento de permutación de respuesta múltiple (MRPP, por sus siglas en inglés) para examinar diferencias en comunidades de macroinvertebrados entre los grupos predefinidos (i) tipos de estaciones de muestreo (referencia e impactada) y (ii) años de salida de campo (2011 y 2012). Siguiendo al MRPP, se utilizó el método de indicador de valor (IV) de Dufrene y Legendre (1997) para identificar especies discriminatorias entre los grupos predeterminados. La significancia de los valores de indicador para cada especie

\begin{tabular}{|c|c|}
\hline 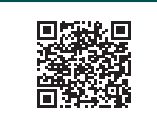 & (c) (i) (5) (2) \\
\hline
\end{tabular}




\section{Revista de CIENCIAS AMBIENTALES Tropical Journal of Environmental Sciences}

Revista de Ciencias Ambientales (Trop J Environ Sci). EISSN: 2215-3896. (Enero-Junio, 2019) . Vol 53(1): 1-22. Dol: http://dx.doi.org/10.15359/rca.53-1.1 Open Access: www.revistas.una.ac.cr/ambientales EMAlL: revista.ambientales@una.ac.cr Lafuente W., Soto L. M., López C., Domínguez-Granda L.

fue examinada con la prueba de aleatorización de Monte Carlo, del cual se corrieron 4999 permutaciones con el software PCORD 6.08.

Se utilizó el análisis de ordenación de escalamiento multidimensional no-métrico (NMDS, por sus siglas en inglés) para evaluar patrones en la estructura de las comunidades de macroinvertebrados para todo el estudio, y se los relacionó con variables ambientales. Para medir distancias ecológicas, se utilizó Sorensen, basado en la presencia/ausencia de datos (Faith et al., 1987). Este análisis fue ejecutado con el software PCORD 6.08, con un valor $\mathrm{r}>0.25$.

Para los análisis efectuados en PCORD 6.08, se eliminaron todas las especies donde solo se había encontrado individuos en una única muestra (cinco especies), y se transformaron los datos utilizando ranking. Esto se hizo con la finalidad de reducir la asimetría y la curtosis, y quedar entre valores de menos uno y más uno.

\section{Resultados}

\subsection{Calidad de agua y sedimentos}

En el Cuadro 1 se muestran los valores de los parámetros fisicoquímicos para todo el estudio.

Cuadro 1. Valores de parámetros fisicoquímicos en cada estación de muestreo durante todo el estudio ( ${ }^{*}$ celdas sin valores)

\begin{tabular}{|c|c|c|c|c|c|c|c|c|c|c|c|c|c|}
\hline 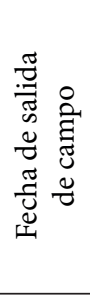 & 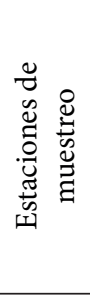 & 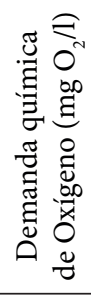 & 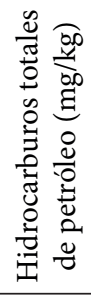 & 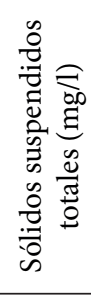 & 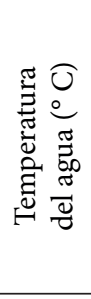 & 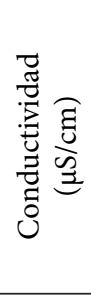 & 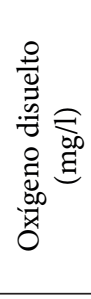 & 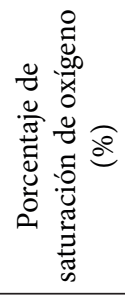 & $\frac{I}{2}$ & 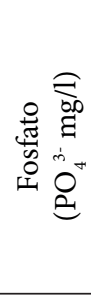 & 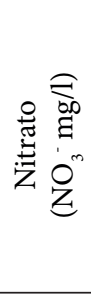 & 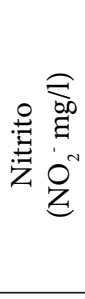 & 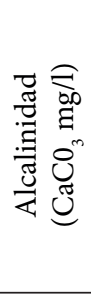 \\
\hline \multirow{11}{*}{ 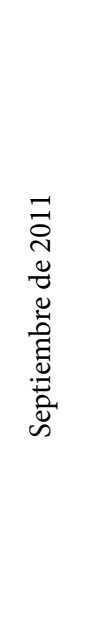 } & E1 & 10.95 & 37 & 20 & 16.3 & 71.6 & 6.95 & 74.1 & 7.60 & 0.046 & 0.38 & 0.19 & 25 \\
\hline & E2 & 12.2 & 63.3 & 12 & 18.5 & 64.5 & 6.82 & 72.4 & 6.08 & 0.046 & 0.64 & 0.13 & 45 \\
\hline & E3 & 9.00 & 50.5 & 10 & 20.3 & 85.6 & 5.62 & 67.0 & 6.39 & 0.046 & 0.54 & 0.06 & 40 \\
\hline & $\mathrm{E} 4$ & 9.20 & 24.0 & 8 & 18.6 & 173.2 & 6.86 & 72.7 & 6.22 & 0.046 & 0.32 & 0.17 & 50 \\
\hline & E5 & 7.95 & 37.2 & 56 & 20.1 & 1.9 & 6.22 & 69.0 & 6.19 & 0.092 & 0.4 & 0.08 & 35 \\
\hline & E6 & 13.95 & 24.0 & 8 & 13.6 & 53.6 & 9.08 & 87.7 & 8.90 & 0.046 & 0.12 & 0.09 & 20 \\
\hline & E7 & 22.45 & 50.2 & 8 & 21.8 & * & 5.00 & 59.3 & 6.87 & 0.092 & 0.64 & 0.09 & 25 \\
\hline & E8 & 24.45 & 37.3 & 10 & 15.1 & 0.1 & 8.26 & 78.4 & 8.51 & 0.046 & 0.34 & 0.32 & 20 \\
\hline & E9 & 22.70 & 24.1 & 44 & 18.4 & 108.1 & 6.98 & 72.6 & 6.50 & 0.092 & 0.92 & 0.16 & 25 \\
\hline & E10 & 5.00 & 37.2 & 38 & 20.5 & 118.7 & 6.68 & 74.5 & 5.69 & 0.092 & 0.76 & 0.15 & 15 \\
\hline & E11 & 14.45 & 24.1 & 42 & 23.9 & * & 5.36 & 65.1 & * & 0.092 & 0.48 & 0.08 & 45 \\
\hline
\end{tabular}

(c)

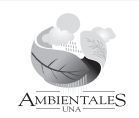




\section{Revista de CIENCIAS AMBIENTALES Tropical Journal of Environmental Sciences}

Revista de Ciencias Ambientales (Trop J Environ Sci). EISSN: 2215-3896. (Enero-Junio, 2019) . Vol 53(1): 1-22. D0l: http://dx.doi.org/10.15359/rca.53-1.1 Open Access: www.revistas.una.ac.cr/ambientales EMAIL: revista.ambientales@una.ac.cr Lafuente W., Soto L. M., López C., Domínguez-Granda L.

\begin{tabular}{|c|c|c|c|c|c|c|c|c|c|c|c|c|c|}
\hline 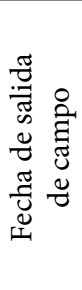 & 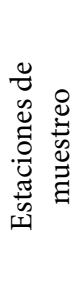 & 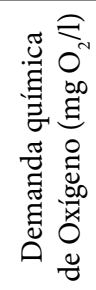 & 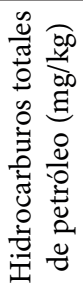 & 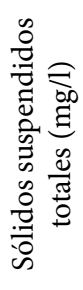 & 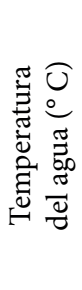 & 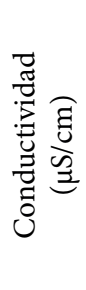 & 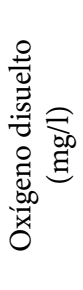 & 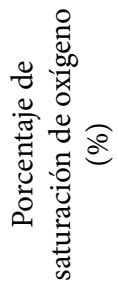 & II & 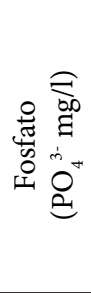 & 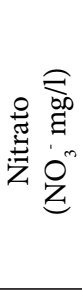 & 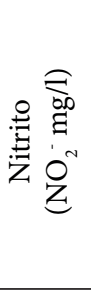 & 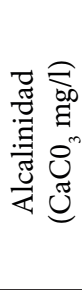 \\
\hline \multirow{11}{*}{ 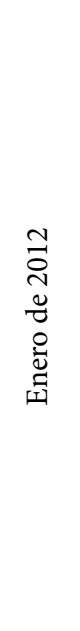 } & E1 & 181.50 & 37.2 & 22 & 14.9 & 125.6 & 8.62 & 85.9 & 9.25 & 0.046 & 1 & 0.24 & 50 \\
\hline & E2 & 116.50 & 11.0 & 26 & 15.8 & 133.1 & 8.06 & 81.7 & 9.21 & 0.092 & 1.24 & 0.29 & 50 \\
\hline & E3 & 170.0 & 23.9 & 16 & 16.5 & 66.1 & 8.01 & 81.6 & 9.08 & 0.046 & 0.44 & 0.05 & 45 \\
\hline & E4 & 80.48 & 24.2 & 54 & 17.3 & 71.5 & 7.91 & 83.0 & 9.28 & 0.250 & 0.84 & 0.11 & 45 \\
\hline & E5 & 210.50 & 37.5 & 70 & 18.2 & 93.7 & 7.56 & 80.3 & 9.11 & 0.092 & 0.9 & 0.32 & 55 \\
\hline & E6 & 189.30 & 11.1 & 20 & 13.1 & 96.8 & 7.36 & 70.1 & 9.81 & 0.046 & 0.22 & 0.60 & 35 \\
\hline & E7 & 54.22 & 11.1 & 12 & 14.5 & 100.7 & 6.99 & 69.6 & 9.64 & 0.046 & 1.42 & 0.20 & 30 \\
\hline & E8 & 35.46 & 11.1 & 12 & 14.4 & 99.4 & 6.96 & 68.1 & 9.46 & 0.046 & 0.88 & 0.08 & 30 \\
\hline & E9 & 233.30 & 37.4 & 22 & 15.8 & 81.6 & 7.77 & 78.9 & 9.05 & 0.046 & 0.58 & 0.10 & 15 \\
\hline & E10 & 71.98 & 11.0 & 16 & 17.4 & 89.2 & 7.57 & 79.7 & 8.22 & 0.046 & 1.82 & 0.11 & 25 \\
\hline & E11 & 38.71 & 11.1 & 24 & 18.2 & 65.7 & 6.63 & 71.2 & 8.71 & 0.092 & 1.08 & 0.09 & 35 \\
\hline
\end{tabular}

\subsection{Macroinvertebrados}

Durante todo el estudio, se recolectaron un total de 4443 individuos pertenecientes a 12 órdenes y 25 familias. Según se puede ver en el Cuadro 2, en el área de estudio, el 76.30 $\%$ de los individuos recolectados pertenecieron al orden Ephemeroptera, seguido del orden Diptera $(11.25 \%)$.

Los datos presentados en el Cuadro 3 indican que el $55.26 \%$ de los individuos recolectados pertenecieron a la familia Baetidae, seguido de la familia Leptophlebiidae (15.77\%).

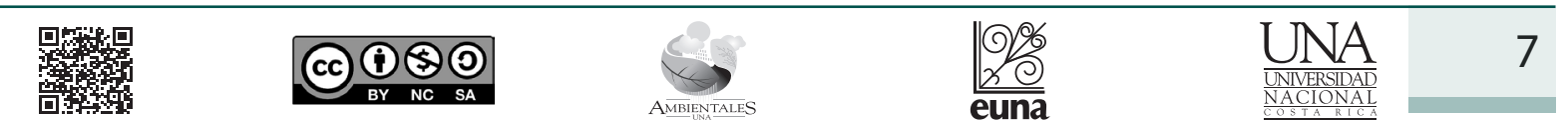




\section{Revista de CIENCIAS AMBIENTALES Tropical Journal of Environmental Sciences}

Revista de Ciencias Ambientales (Trop J Environ Sci). EISSN: 2215-3896. (Enero-Junio, 2019) . Vol 53(1): 1-22. D0l: http://dx.doi.org/10.15359/rca.53-1.1 Open Access: www.revistas.una.ac.cr/ambientales EMAlL: revista.ambientales@una.ac.cr Lafuente W., Soto L. M., López C., Domínguez-Granda L.

Cuadro 2. Número de macroinvertebrados a nivel de orden en cada estación de muestreo durante todo el estudio

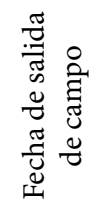

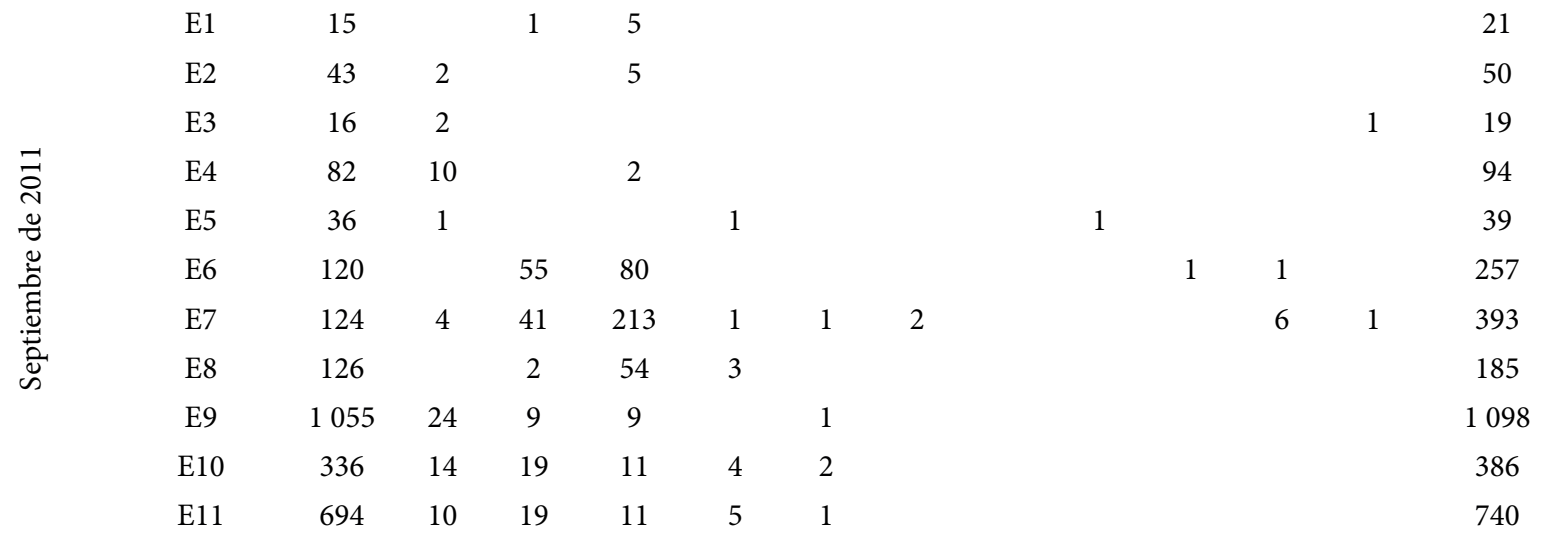

\begin{tabular}{|c|c|c|c|c|c|c|c|c|c|c|c|c|c|}
\hline \multirow{11}{*}{ 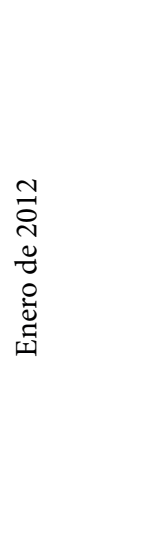 } & 8 & & 1 & & 1 & & 1 & & & & & & 11 \\
\hline & 11 & 4 & 2 & & & & & & & & & & 17 \\
\hline & 78 & 5 & 1 & 4 & 6 & & & & & & & & 94 \\
\hline & 13 & 2 & & 1 & & & 1 & & & & & & 17 \\
\hline & 5 & 2 & & & 1 & & & & & & & & 8 \\
\hline & 60 & 2 & 22 & 46 & 14 & 2 & & & & & 1 & & 147 \\
\hline & 53 & 6 & 40 & 41 & 17 & 1 & 2 & & & & & & 160 \\
\hline & 5 & 1 & 1 & 8 & & & & & & & & & 15 \\
\hline & 97 & 2 & 4 & 1 & 4 & & & & & & & & 108 \\
\hline & 270 & 44 & 34 & 1 & 26 & 3 & & 1 & & & & & 379 \\
\hline & 143 & 6 & 47 & 8 & 1 & & & & & & & & 205 \\
\hline $\begin{array}{l}\text { centaje de individuos } \\
\text { orden }(\%)\end{array}$ & 76.30 & 3.17 & 6.71 & 11.25 & 1.89 & 0.25 & 0.14 & 0.02 & 0.02 & 0.02 & 0.18 & 0.05 & \\
\hline $\begin{array}{l}\text { mero total de } \\
\text { ividuos }\end{array}$ & 3390 & 141 & 298 & 500 & 84 & 11 & 6 & 1 & 1 & 1 & 8 & 2 & 4443 \\
\hline
\end{tabular}

(c) (1) $\$($ )




\section{Revista de $=$ CIENCIAS AMBIENTALES Tropical Journal of Environmental Sciences}

Revista de Ciencias Ambientales (Trop J Environ Sci). EISSN: 2215-3896. (Enero-Junio, 2019) . Vol 53(1): 1-22.

D0l: http://dx.doi.org/10.15359/rca.53-1.1

Open Access: www.revistas.una.ac.cr/ambientales

EMAlL: revista.ambientales@una.ac.cr Lafuente W., Soto L. M., López C., Domínguez-Granda L.

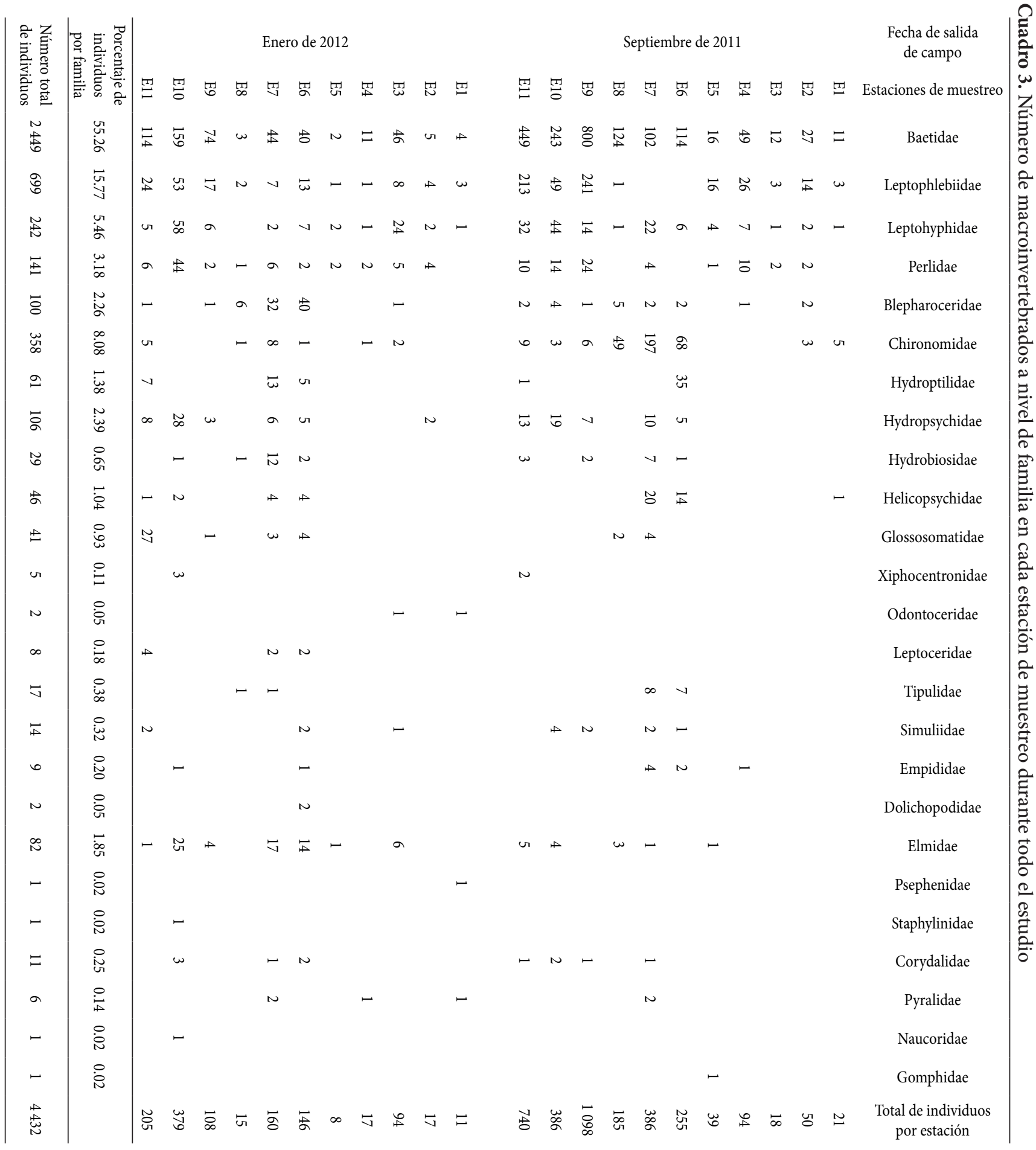




\section{Revista de CIENCIAS AMBIENTALES Tropical Journal of Environmental Sciences}

Revista de Ciencias Ambientales (Trop J Environ Sci). EISSN: 2215-3896. (Enero-Junio, 2019) . Vol 53(1): 1-22. D0l: http://dx.doi.org/10.15359/rca.53-1.1 Open Access: www.revistas.una.ac.cr/ambientales EMAlL: revista.ambientales@una.ac.c Lafuente W., Soto L. M., López C., Domínguez-Granda L.

\subsection{Métricas bióticas}

Los resultados de las medidas de abundancia, diversidad, riqueza de taxa, medidas de composición e índices bióticos aplicadas a las comunidades de macroinvertebrados bentónicos de los ríos Santa Rosa, Quijos y Coca, para las dos salidas de campo, se encuentran en los Apéndices $1,2,3$ y 4 .

Análisis de correlación de Spearman entre la riqueza de taxa de macroinvertebrados bentónicos y las variables ambientales registradas durante todo el estudio determinaron que los taxa EPT $(r=-0.5002 ; \mathrm{p}=0,0177)$ y Trichoptera $(\mathrm{r}=-0.5060 ; \mathrm{p}=0.0162)$ estuvieron correlacionados de manera significativa con los hidrocarburos totales de petróleo.

Análisis similares fueron realizados entre el número de familias dentro de cada orden y las variables ambientales. En la Figura 2 se indica que la familia Elmidae (Coleoptera) mostró una correlación negativa con los hidrocarburos totales de petróleo $(r=-0.6982 ; \mathrm{p}=0.0116)$.

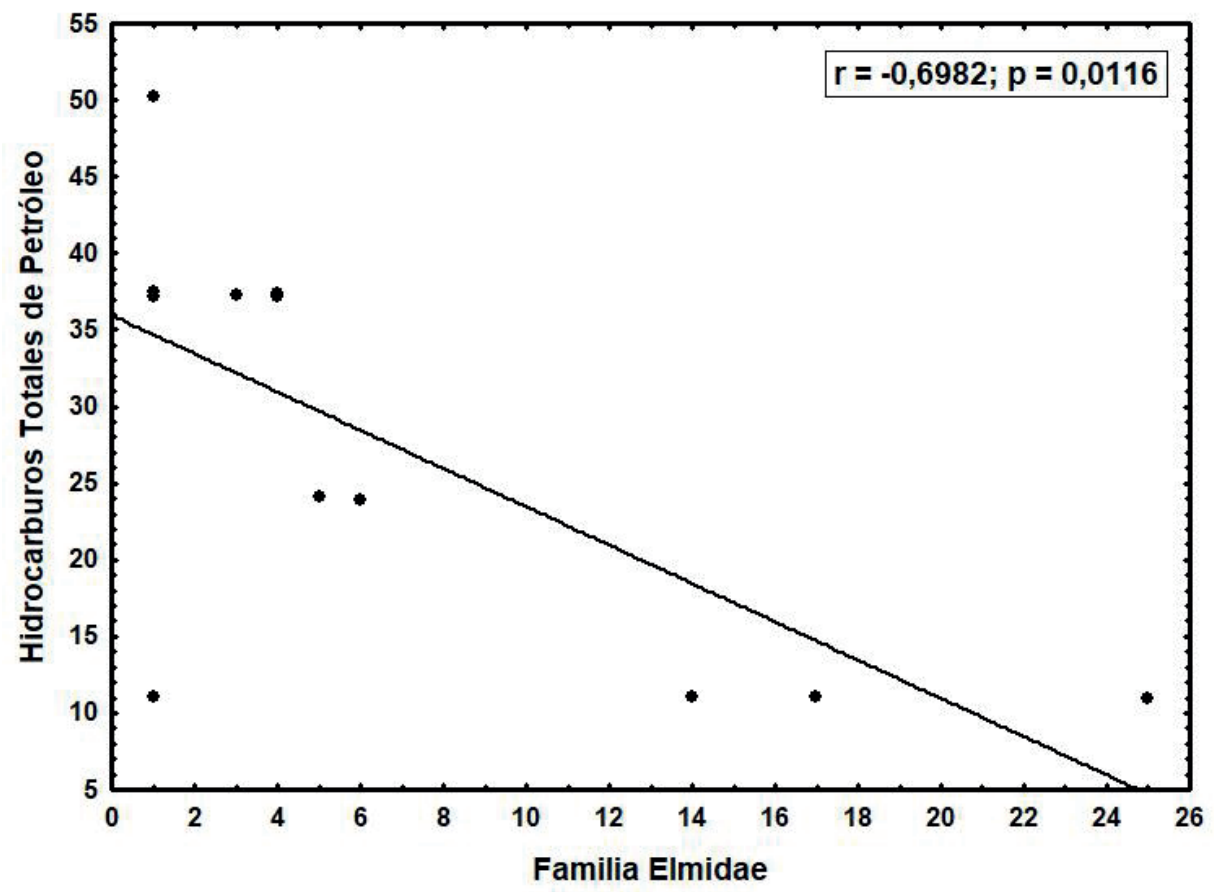

Figura 2. Relación de hidrocarburos totales de petróleo con la familia Elmidae (Coleoptera) durante todo el estudio.

El análisis de correlación de Spearman entre los índices bióticos y las variables ambientales registradas durante todo el estudio determinó una correlación negativa entre el índice SIGNAL2 y los hidrocarburos totales de petróleo $(r=-0.5886 ; \mathrm{p}=0.0040)$, como se indica en la Figura 3 .

\section{口我吅




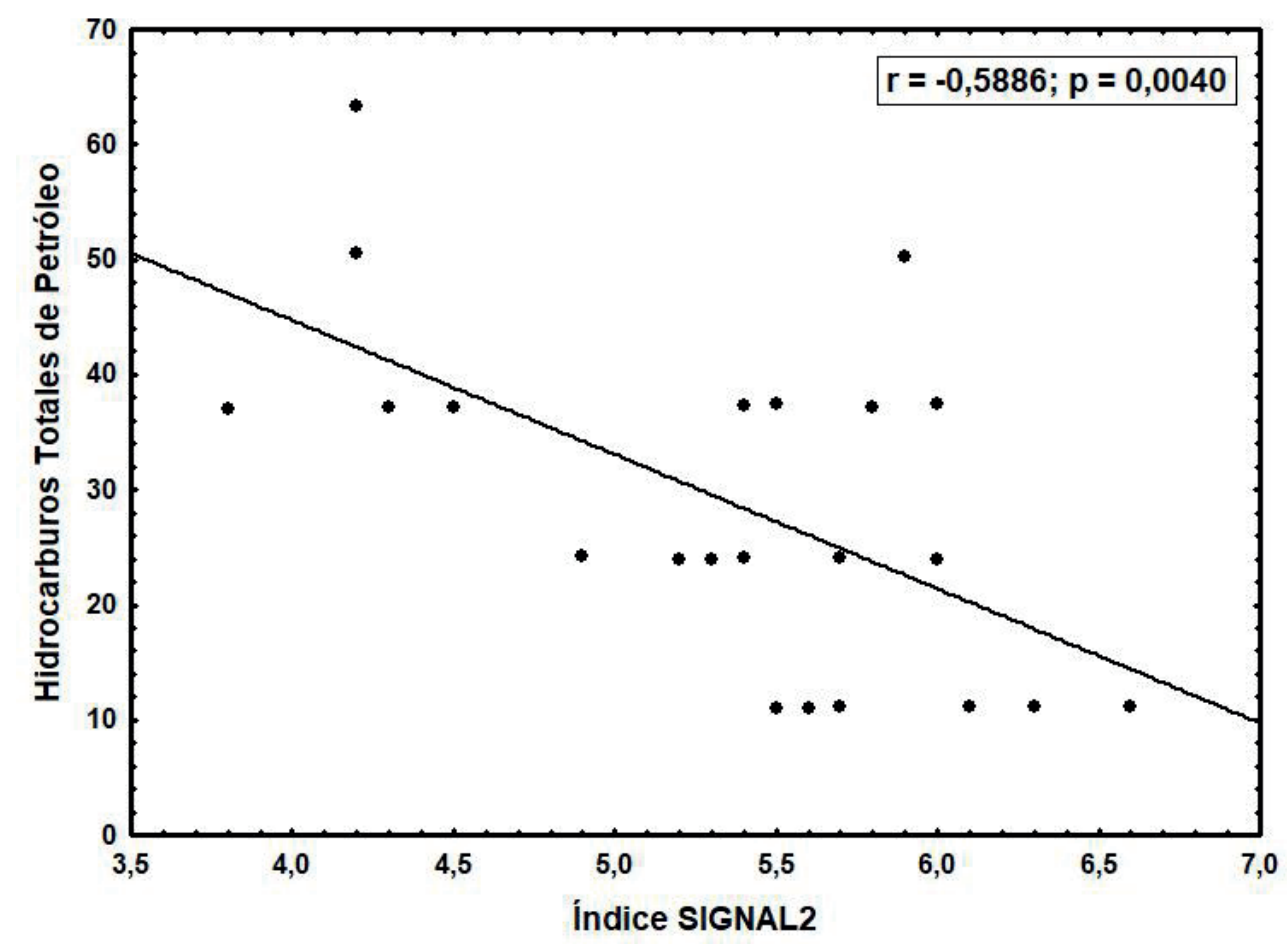

Figura 3. Relación de hidrocarburos totales de petróleo con el índice SIGNAL2 durante todo el estudio.

\subsection{Análisis MRPP, IV y NMDS}

El análisis de agrupación MRPP determinó una diferencia entre las comunidades de macroinvertebrados de estaciones de referencia e impactadas del año 2011, y entre las comunidades de macroinvertebrados de estaciones de referencia año 2011 y puntos impactados año $2012(\mathrm{~A}=$ $0.08, \mathrm{p}=0.02)$. El análisis del método IV estableció especies que son indicadoras de estaciones de referencia del año 2011 (Helicopsychidae, $\mathrm{p}=0.02$; Chironomidae, $\mathrm{p}=0.0002$ ), y una especie indicadora de estación impactada del año 2012 (Leptophlebiidae, $\mathrm{p}=0.0006$ ).

El análisis de ordenación NMDS sugirió usar tres dimensiones. El eje uno separa las comunidades de macroinvertebrados de las estaciones de referencia del año 2011 y las comunidades de macroinvertebrados de las estaciones impactadas del año 2011 y las comunidades del año 2012, donde la alcalinidad $(r=0.42)$ es la variable que explica de mejor manera esta ordenación. Hidrocarburos totales de petróleo $(r=0.45)$ es la variable que mejor se relaciona con el eje dos, y temperatura $(r=0.53)$ se relaciona de mejor manera con el eje 3 , como se indica en las Figuras 4,5 y 6.

(cc)




\section{Revista de Desde
since 1980 CIENCIAS AMBIENTALES Tropical Journal of Environmental Sciences}

Revista de Ciencias Ambientales (Trop J Environ Sci). EISSN: 2215-3896.

(Enero-Junio, 2019) . Vol 53(1): 1-22.

D0l: http://dx.doi.org/10.15359/rca.53-1.1

Open Access: www.revistas.una.ac.cr/ambientales

EMAIL: revista.ambientales@una.ac.cr Lafuente W., Soto L. M., López C., Domínguez-Granda L.

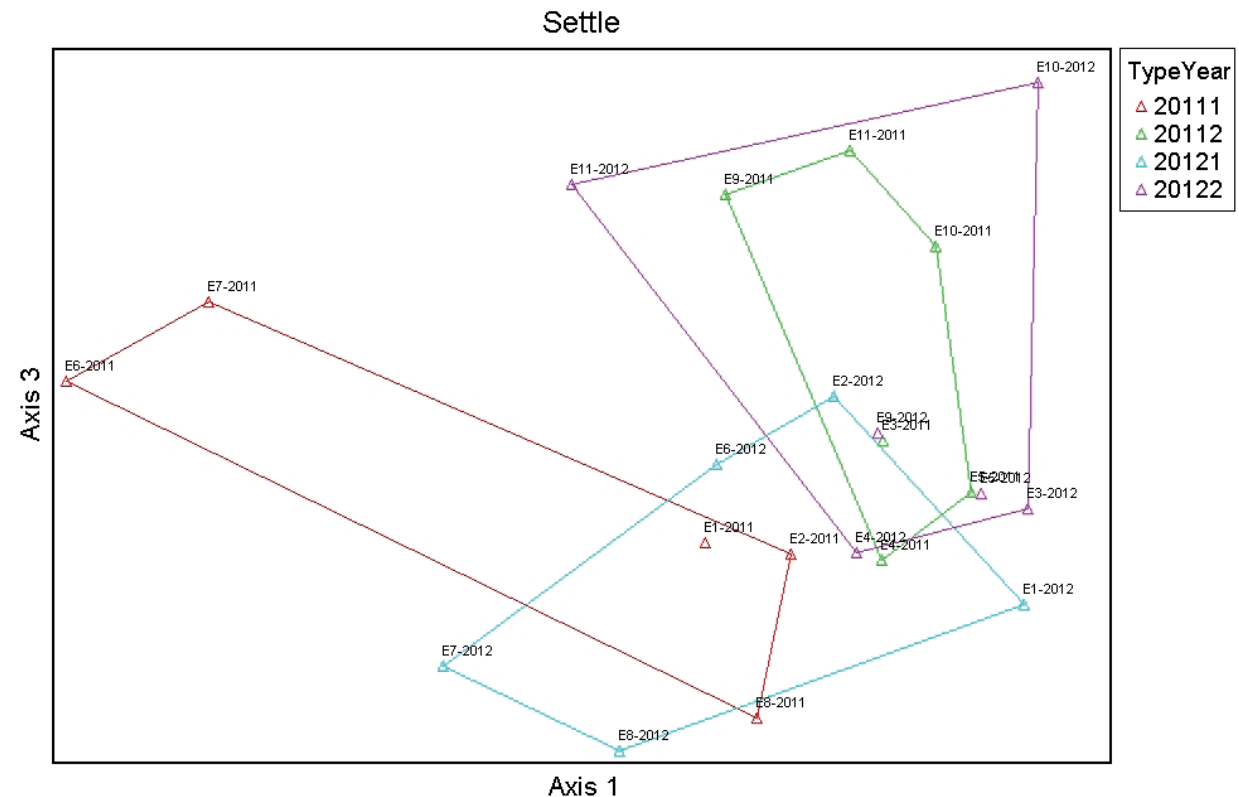

Figura 4. Análisis de ordenación NMDS entre las comunidades de macroinvertebrados de las estaciones de referencia e impactadas durante todo el estudio. El eje uno se relaciona con $\mathrm{CaCO}_{3}(\mathrm{r}=0.42)$.

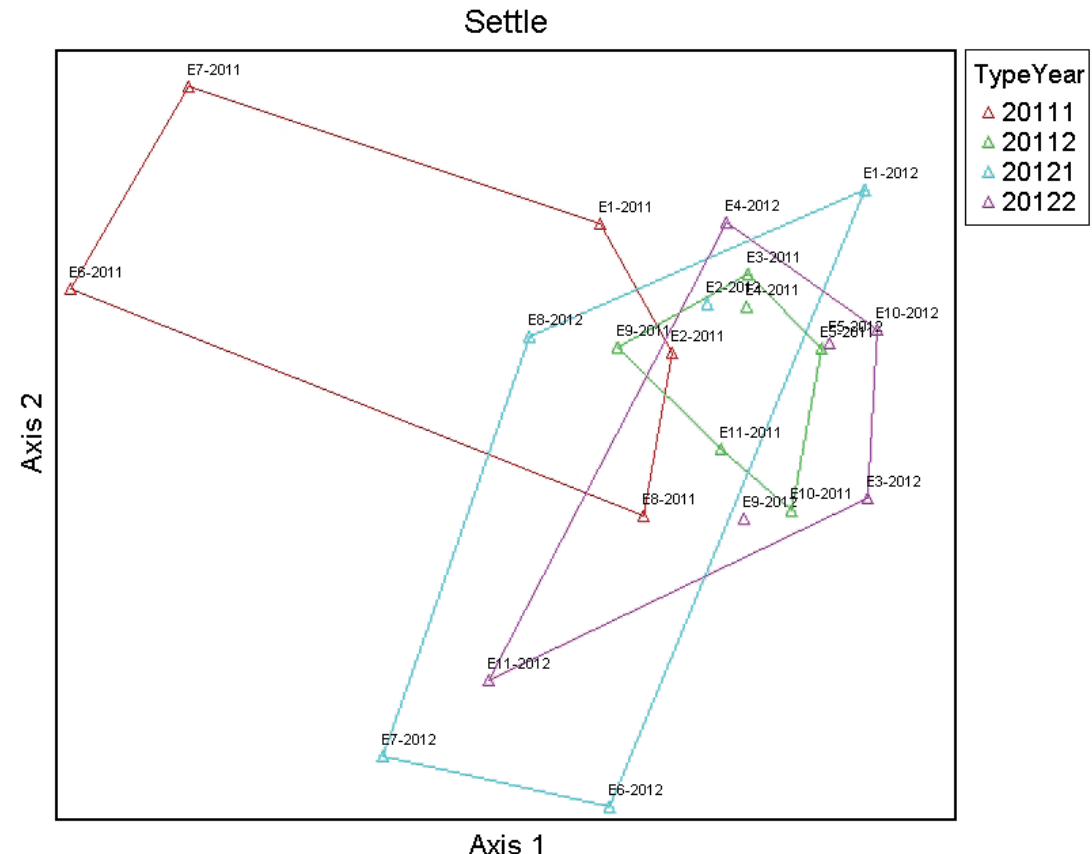

Figura 5. Análisis de ordenación NMDS entre las comunidades de macroinvertebrados de las estaciones de referencia e impactadas durante todo el estudio. El eje dos se relaciona con HTP $(r=0.45)$.
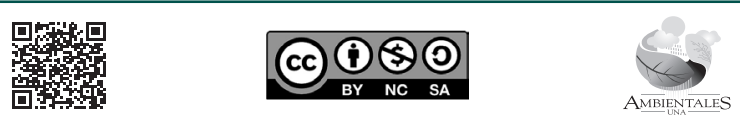


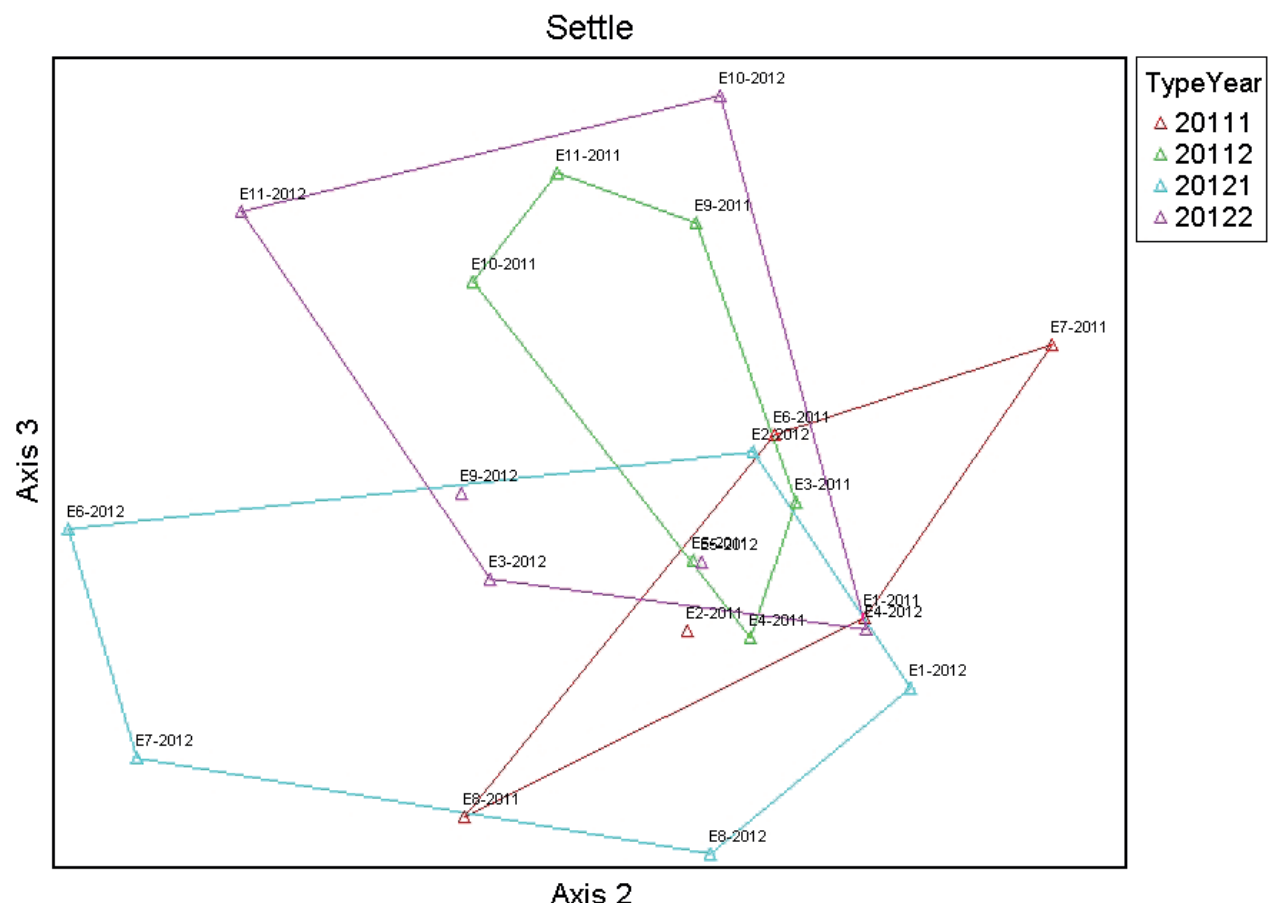

Figura 6. Análisis de ordenación NMDS entre las comunidades de macroinvertebrados de las estaciones de referencia e impactadas durante todo el estudio. El eje tres se relaciona con temperatura $(r=0.45)$.

\section{Discusión}

Relativamente pocos estudios describen la composición de las comunidades de macroinvertebrados bentónicos de ríos afectados por derrames de hidrocarburos, particularmente en América del Sur. Las variables ambientales evaluadas, entre ellas los hidrocarburos totales de petróleo, podrían inducir diferentes respuestas en la composición de la fauna acuática a las condiciones ambientales afectadas por la presencia del hidrocarburo en el ecosistema acuático. Esta alteración es compleja debido a factores tales como volumen derramado, composición química del petróleo, impactos previos, condiciones climáticas, morfología e hidráulica del río receptor, y las características ecológicas propias de la biota asociada (Crunkilton y Duchrow, 1990; Masnik et al., 1976; Parker et al., 1976).

La relación inversa entre los taxa EPT y Trichoptera con los HTP es consistente con otros estudios que señalan una inmediata reducción en la densidad y riqueza taxonómica de invertebrados tanto de manera temporal como espacial (Barton y Wallace, 1979; Bugbee y Walter, 1973; Rosenberg y Wiens, 1976). Crunkilton y Duchrow (1990) encontraron que las especies de Ephemeroptera, Plecoptera y Trichoptera estuvieron ausentes nueve meses después de un derrame de 1.5 millones de litros de crudo doméstico sobre el río Asher, Estados Unidos. En nuestros

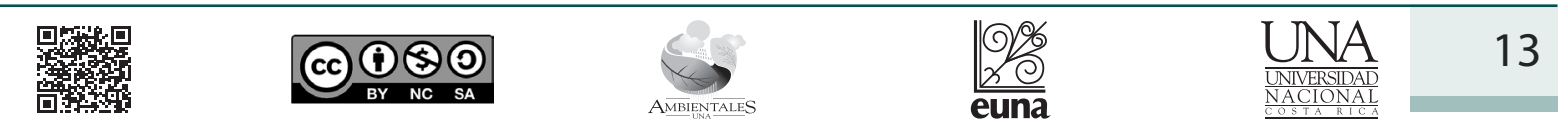




\section{Revista de CIENCIAS AMBIENTALES Tropical Journal of Environmental Sciences}

Revista de Ciencias Ambientales (Trop J Environ Sci). EISSN: 2215-3896. (Enero-Junio, 2019) . Vol 53(1): 1-22. D0l: http://dx.doi.org/10.15359/rca.53-1.1 Open Access: www.revistas.una.ac.cr/ambientales EMAlL: revista.ambientales@una.ac.cr Lafuente W., Soto L. M., López C., Domínguez-Granda L.

resultados se observó un incremento en el número de Ephemeroptera en los sitios impactados, lo cual pudiera explicarse por las diferencias en la composición específica de ese grupo biológico. Además de eso, podrían sugerir una mayor capacidad de recuperación de este tipo de organismos en los trópicos.

Poulton et al. (1997) determinaron que los grupos de macroinvertebrados de aguas de remanso más severamente afectados fueron los taxones Trichoptera y Ephemeroptera, año y medio después de un derrame de 3.3 millones de litros de crudo sobre un río grande en Misuri, Estados Unidos. Lytle y Peckarsky (2001) reportaron una reducción del 50 \% en la riqueza de taxones sensibles a hidrocarburos al menos cinco kilómetros río abajo del derrame de 26500 litros de diésel sobre un tributario del río Cayuga, Estados Unidos, después de 15 meses de ocurrido el derrame. A pesar de que en nuestro estudio se encontró una relación negativa entre el taxa Trichoptera y los HTP, la abundancia de este grupo en las estaciones de referencia fue muy baja, y la disminución de su abundancia en el río Coca con respecto al Quijos puede deberse a cualquier otro factor.

A pesar de que se encontró una relación negativa entre la familia Elmidae y los HTP, la abundancia de este grupo en las estaciones de referencia fue muy baja y, por tanto, puede ser otro factor diferente a los HTP el responsable de esa correlación.

La relación inversa entre el índice SIGNAL2 con los HTP no ha sido reportada en otros estudios. Sin embargo, Smith et al. (2010) determinaron la recuperación de las comunidades de macroinvertebrados de un río urbano en Wiltshire, Reino Unido, afectado por un derrame de 9800 litros de diésel, mediante el uso del índice BMWP. A pesar de que el índice BMWP fue diseñado para contaminación orgánica (Hawkes, 1997), su coincidencia con datos de contaminación por hidrocarburos pudo deberse a la disminución general de la biodiversidad.

No obstante, las diferencias entre las comunidades de macroinvertebrados de estaciones de referencia e impactadas del año 2011, y entre las comunidades de estaciones de referencia año 2011 y puntos impactados año 2012 podrían indicar que las agrupaciones de fauna acuática son distintas debido a diferencias en el orden del río (Vannote et al., 1980).

Las ordenaciones NMDS de las comunidades de macroinvertebrados en las diferentes dimensiones, agrupadas de acuerdo con las variables tipos de estaciones (referencia e impactada) y relacionadas con $\mathrm{CaCO}_{3}$, HTP y temperatura no han sido registradas en otros estudios. Sin embargo, Vinson et al. (2008) reportaron que las ordenaciones NMDS de macroinvertebrados de un humedal en Gabón, impactado por actividades de extracción de petróleo desde la década del 50, no se agruparon de acuerdo con la contaminación del sitio, sino con la variable de permanencia del agua. A pesar de que los ríos Santa Rosa, Quijos y Coca son permanentes, el análisis NMDS funcionó al ordenar las comunidades de macroinvertebrados con el eje dos que se relaciona con HTP.

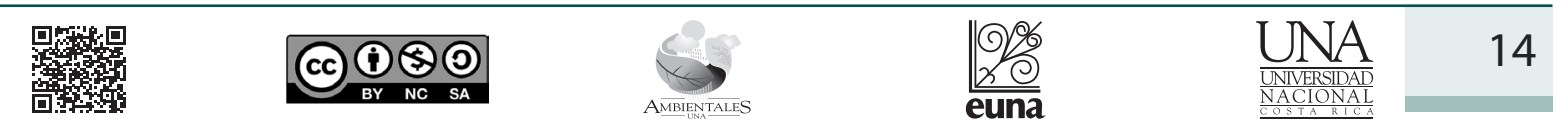




\section{Revista de CIENCIAS AMBIENTALES Tropical Journal of Environmental Sciences}

Revista de Ciencias Ambientales (Trop J Environ Sci). EISSN: 2215-3896. (Enero-Junio, 2019) . Vol 53(1): 1-22. D0l: http://dx.doi.org/10.15359/rca.53-1.1 Open Access: www.revistas.una.ac.cr/ambientales EMAIL: revista.ambientales@una.ac.c Lafuente W., Soto L. M., López C., Domínguez-Granda L.

\section{Conclusiones}

En general, el presente estudio sugiere que la comunidad de macroinvertebrados de los ríos Santa Rosa, Quijos, y Coca, aún después de 31 meses de ocurrido el derrame de hidrocarburo, no se ha recuperado de manera completa, de acuerdo con las correlaciones negativas entre los HTP y los taxa EPT, Trichoptera y Elmidae, y el índice SIGNAL2, exhibidos durante todo el estudio. No obstante, la heterogeneidad espacial y temporal en las comunidades de macroinvertebrados de todas las estaciones de muestreo sugiere que factores distintos a los HTP pudieron ser responsables de su variada composición.

Se recomienda la realización de monitoreos de ríos afectados por derrames de hidrocarburos haciendo uso de la comunidad de macroinvertebrados bentónicos, desde el comienzo del derrame hasta un mínimo de cinco años después de haber culminado las acciones de remediación ambiental. Se espera, de esta forma, comprender de mejor manera el proceso de recuperación del ecosistema fluvial. Adicionalmente a esto, se sugiere evaluar el impacto en estas comunidades bióticas, por los métodos de remediación aplicados en regiones tropicales.

Finalmente, futuros estudios podrían determinar el comportamiento del petróleo y los efectos letales y subletales de sus principales componentes tanto in situ como en condiciones de laboratorio, hasta el nivel taxonómico de especie, en especial para los taxones sensibles a los bajos niveles de HTP reportados en esta investigación.

\section{Agradecimientos}

Este estudio fue financiado por el Centro de Investigación Científica y Tecnológica (CICYT) de la Escuela Superior Politécnica del Litoral (ESPOL) (proyecto semilla con código PTMA-10009). Los análisis químicos y biológicos fueron realizados en el laboratorio de medio ambiente y en el laboratorio de aguas de la Facultad de Ciencias Naturales y Matemáticas (FCNM), y en el laboratorio de bentos de la Facultad de Ingeniería Marítima, Ciencias Biológicas, Oceánicas y Recursos Naturales (FIMCBOR), de ESPOL, respectivamente. Agradecemos al doctor José Marín y a la doctora Mariela González, por los análisis estadísticos, y a la doctora María Fernanda Calderón, por la elaboración del mapa del área de estudio. Finalmente, agradecemos a la Revista y a las personas revisoras anónimas por sus valiosos comentarios que enriquecieron la versión final del manuscrito.

\section{Referencias}

Alemán, M. Á., Branch, E. I., Bermeo, R., SA, O. E., Mendizábal, A., \& Loon, W. (2010). Successful Social Environmental Management Model, Implemented in Ecuador to Overcome Impacts From a Heavy Crude Oil Spill. 2010 8th International Pipeline Conference (pp. 149158). American Society of Mechanical Engineers. https://doi.org/10.1115/IPC2010-31179

American Public Health Association [APHA]. (2005). Standard Methods for the Examination of Water and Wastewater. 21st ed. American Public Health Association, Washington DC, 1220p.

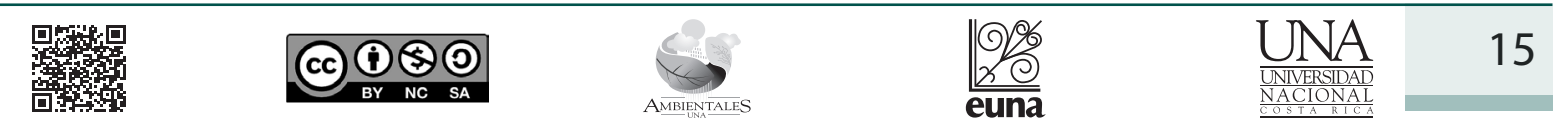




\section{Revista de CIENCIAS AMBIENTALES Tropical Journal of Environmental Sciences}

Revista de Ciencias Ambientales (Trop J Environ Sci). EISSN: 2215-3896. (Enero-Junio, 2019) . Vol 53(1): 1-22. D0l: http://dx.doi.org/10.15359/rca.53-1.1 Open Access: www.revistas.una.ac.cr/ambientales EMAIL: revista.ambientales@una.ac.c Lafuente W., Soto L. M., López C., Domínguez-Granda L.

Araújo, C. V., Moreira-Santos, M., Sousa, J. P., Ochoa-Herrera, V., Encalada, A. C., \& Ribeiro, R. (2014). Contaminants as habitat disturbers: PAH-driven drift by Andean paramo stream insects. Ecotoxicology and environmental safety, 108, 89-94. https://doi.org/10.1016/j. ecoenv.2014.06.034

Barton, D. R., \& Wallace, R. R. (1979). The effects of an experimental spillage of oil sands tailings sludge on benthic invertebrates. Environmental Pollution (1970), 18(4), 305-312. https://doi. org/10.1016/0013-9327(79)90025-9

Bugbee, S. L., \& Walter, C. M. (1973). The response of macroinvertebrates to gasoline pollution in a mountain stream. International Oil Spill Conference (Vol. 1973, No. 1, pp. 725-731). American Petroleum Institute. https://doi.org/10.7901/2169-3358-1973-1-725

Couceiro, S. R., Hamada, N., Ferreira, R. L., Forsberg, B. R., \& Da silva, J. O. (2007). Domestic sewage and oil spills in streams: effects on edaphic invertebrates in flooded forest, Manaus, Amazonas, Brazil. Water, air, and soil pollution, 180(1-4), 249-259. https://doi.org/10.1007/ s11270-006-9267-y

Cueva Sánchez, M. G. (2013). Efectos del derrame de petróleo sobre la dinámica ecológica de las comunidades zooplanctónicas de la laguna de Papallacta (Bachelor's tesis). Universidad Internacional del Ecuador. Recuperado de http://repositorio.uide.edu.ec/bitstream/37000/298/1/T-UIDE-0277.pdf

Crunkilton, R. L., \& Duchrow, R. M. (1990). Impact of a massive crude oil spill on the invertebrate fauna of a Missouri Ozark stream. Environmental Pollution, 63(1), 13-31. https://doi. org/10.1016/0269-7491(90)90100-Q

De Pauw, N., \& Vanhooren, G. (1983). Method for biological quality assessment of watercourses in Belgium. Hydrobiologia, 100(1), 153-168. https://doi.org/10.1007/BF00027428

Domínguez, E., \& Fernández, H. R. (2009). Macroinvertebrados bentónicos sudamericanos: Sistemática y biología. Fundación Miguel Lillo, Tucumán, Argentina, 656.

Dufrêne, M., \& Legendre, P. (1997). Species assemblages and indicator species: the need for a flexible asymmetrical approach. Ecological monographs, 67(3), 345-366. https://doi.org/10.1890/0012-9615(1997)067[0345:SAAIST]2.0.CO;2 https://doi.org/10.2307/2963459

Faith, D. P., Minchin, P. R., \& Belbin, L. (1987). Compositional dissimilarity as a robust measure of ecological distance. Vegetatio, 69(1-3), 57-68. https://doi.org/10.1007/BF00038687

Harrel, R. C. (1985). Effects of a crude oil spill on water quality and macrobenthos of a southeast Texas stream. Hydrobiologia, 124(3), 223-228. https://doi.org/10.1007/BF00015238

Hawkes, H. A. (1998). Origin and development of the biological monitoring working party score system. Water Research, 32(3), 964-968. https://doi.org/10.1016/S0043-1354(97)00275-3

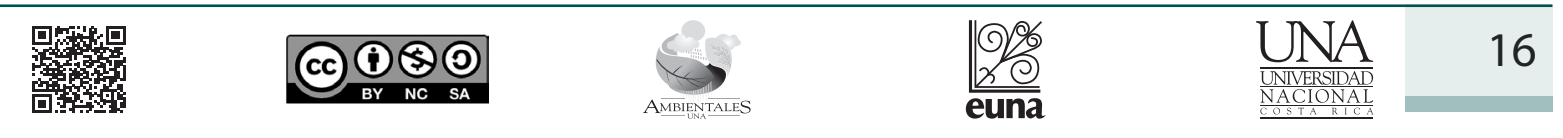




\section{Revista de CIENCIAS AMBIENTALES Tropical Journal of Environmental Sciences}

Revista de Ciencias Ambientales (Trop J Environ Sci). EISSN: 2215-3896. (Enero-Junio, 2019) .Vol 53(1): 1-22. Dol: http://dx.doi.org/10.15359/rca.53-1.1 Open Access: www.revistas.una.ac.cr/ambientales EMAlL: revista.ambientales@una.ac.cr Lafuente W., Soto L. M., López C., Domínguez-Granda L.

Lytle, D. A., \& Peckarsky, B. L. (2001). Spatial and temporal impacts of a diesel fuel spill on stream invertebrates. Freshwater Biology, 46(5), 693-704. https://doi. org/10.1046/j.1365-2427.2001.00695.x

Masnik, M. T., Stauffer, J. R., Hocutt, C. H., \& Wilson, J. H. (1976). The effects of an oil spill on the macroinvertebrates and fish in a small southwestern Virginia creek. Journal of Environmental Science \& Health Part A, 11(4-5), 281-296. https://doi.org/10.1080/10934527609385771

Mendizábal, A., Samaniego, R., \& Aleman, M. A. (2012). Three years later-conclusion of a successful social-environmental management model to overcome the impacts of a heavy crude oil spill. 2012 9th International Pipeline Conference (pp. 263-274). American Society of Mechanical Engineers. https://doi.org/10.1115/IPC2012-90009

Molina Santos, M. G. (2013). Efectos del derrame de petróleo sobre la comunidad Fitoplanctónica de la laguna de Papallacta y sus principales afluentes (Tesis de bachillerato) UIDE, Quito. Recuperado de http://repositorio.uide.edu.ec/bitstream/37000/301/1/T-UIDE-0280.pdf

Ort, M. P., Finger, S. E., \& Jones, J. R. (1995). Toxicity of crude oil to the mayfly, Hexagenia bilineata (Ephemeroptera: Ephemeridae). Environmental Pollution, 90(1), 105-110. https://doi. org/10.1016/0269-7491(94)00085-R

Parker, B. L., Brammer, J. D., Whalon, M. E., \& Berry, W. O. (1976). Chronic oil contamination and aquatic organisms with emphasis on Diptera: status and bibliography. JAWRA Journal of the American Water Resources Association, 12(2), 291-305. https://doi.or$\mathrm{g} / 10.1111 / \mathrm{j} .1752-1688.1976 . t b 02680 . \mathrm{x}$

Paumen, M. L., Borgman, E., Kraak, M. H., Van Gestel, C. A., \& Admiraal, W. (2008). Life cycle responses of the midge Chironomus riparius to polycyclic aromatic compound exposure. Environmental pollution, 152(1), 225-232. https://doi.org/10.1016/j.envpol.2007.04.027

Pettigrove, V., \& Hoffmann, A. (2005). Effects of long-chain hydrocarbon-polluted sediment on freshwater macroinvertebrates. Environmental Toxicology and Chemistry, 24(10), 2500-2508. https://doi.org/10.1897/05-018R.1

Poulton, B. C., Finger, S. E., \& Humphrey, S. A. (1997). Effects of a crude oil spill on the benthic invertebrate community in the Gasconade River, Missouri. Archives of Environmental Contamination and Toxicology, 33(3), 268-276. https://doi.org/10.1007/s002449900253

Roldán, G. (1988). Guía para el estudio de los macroinvertebrados acuáticos del Departamento de Antioquia. Universidad de Antioquia, Fondo FEN, Medellín.

Rosenberg, D. M., \& Wiens, A. P. (1976). Community and species responses of Chironomidae (Diptera) to contamination of fresh waters by crude oil and petroleum products, with special

\begin{tabular}{|c|c|c|}
\hline 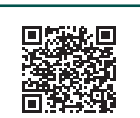 & (c) (1) () (2) & 17 \\
\hline
\end{tabular}




\section{Revista de CIENCIAS AMBIENTALES Tropical Journal of Environmental Sciences}

Revista de Ciencias Ambientales (Trop J Environ Sci). EISSN: 2215-3896. (Enero-Junio, 2019) . Vol 53(1): 1-22. D0l: http://dx.doi.org/10.15359/rca.53-1.1 Open Access: www.revistas.una.ac.cr/ambientales EMAIL: revista.ambientales@una.ac.cr Lafuente W., Soto L. M., López C., Domínguez-Granda L.

reference to the Trail River, Northwest Territories. Journal of the Fisheries Board of Canada, 33(9), 1955-1963. https://doi.org/10.1139/f76-249

Smith, P., Snook, D., Muscutt, A., \& Smith, A. (2010). Effects of a diesel spill on freshwater macroinvertebrates in two urban watercourses, Wiltshire, UK. Water and Environment Journal, 24(4), 249-260. https://doi.org/10.1111/j.1747-6593.2009.00168.x

Strahler, A. N. (1957). Quantitative analysis of watershed geomorphology. Eos, Transactions American Geophysical Union, 38(6), 913-920. https://doi.org/10.1029/TR038i006p00913

Vannote, R. L., Minshall, G. W., Cummins, K. W., Sedell, J. R., \& Cushing, C. E. (1980). The river continuum concept. Canadian journal of fisheries and aquatic sciences, 37(1), 130-137. https://doi.org/10.1139/f80-017

Vinson, M. R., Dinger, E. C., Kotynek, J., \& Dethier, M. (2008). Effects of oil pollution on aquatic macroinvertebrate assemblages in Gabon wetlands. African Journal of Aquatic Science, 33(3), 261-268. https://doi.org/10.2989/AJAS.2008.33.3.9.621

Zar, J. H. (1996). Biostatistical analysis. Prentice-Hall International. Inc., London. 


\section{Revista de Desde
Since 1980 CIENCIAS AMBIENTALES Tropical Journal of Environmental Sciences}

Revista de Ciencias Ambientales (Trop J Environ Sci). EISSN: 2215-3896. (Enero-Junio, 2019) . Vol 53(1): 1-22. D0l: http://dx.doi.org/10.15359/rca.53-1.1 Open Access: www.revistas.una.ac.cr/ambientales EMAlL: revista.ambientales@una.ac.c Lafuente W., Soto L. M., López C., Domínguez-Granda L.

\section{Apéndices}

Apéndice 1. Índices bióticos calculados para cada estación de muestreo para el año 2011

\begin{tabular}{|c|c|c|c|c|c|c|c|c|c|c|c|}
\hline Código de estación & E1 & $\mathrm{E} 2$ & E3 & $\mathrm{E} 4$ & E5 & E6 & E7 & E8 & E9 & E10 & E11 \\
\hline \multicolumn{12}{|c|}{ Medidas de abundancia } \\
\hline N. ${ }^{\circ}$ de individuos & 22 & 50 & 19 & 94 & 39 & 257 & 393 & 185 & 1098 & 386 & 740 \\
\hline \multicolumn{12}{|l|}{ Medidas de diversidad } \\
\hline Margalef & 1.6 & 1.3 & 1.4 & 1.1 & 1.4 & 2.2 & 2.7 & 1.1 & 1.3 & 1.5 & 1.7 \\
\hline Shannon-Wiener & 1.4 & 1.2 & 1.1 & 1.2 & 1.2 & 1.6 & 1.6 & 0.9 & 0.8 & 1.3 & 1.1 \\
\hline Simpson & 0.7 & 0.6 & 0.6 & 0.6 & 0.7 & 0.7 & 0.7 & 0.5 & 0.4 & 0.6 & 0.5 \\
\hline Eveness & 0.8 & 0.7 & 0.7 & 0.7 & 0.7 & 0.6 & 0.6 & 0.5 & 0.3 & 0.6 & 0.4 \\
\hline \multicolumn{12}{|c|}{ Medidas de riqueza de taxa } \\
\hline Número de taxa & 6 & 6 & 5 & 6 & 6 & 13 & 17 & 7 & 10 & 10 & 12 \\
\hline N. ${ }^{\circ}$ EPT taxa & 4 & 4 & 4 & 4 & 4 & 6 & 7 & 4 & 6 & 5 & 8 \\
\hline N. ${ }^{\circ}$ Ephemeroptera taxa & 3 & 3 & 3 & 3 & 3 & 2 & 2 & 3 & 3 & 3 & 3 \\
\hline N. ${ }^{\circ}$ Trichoptera taxa & 1 & 0 & 0 & 0 & 0 & 4 & 4 & 1 & 2 & 1 & 4 \\
\hline N. ${ }^{\circ}$ Diptera Taxa & 1 & 2 & 0 & 2 & 0 & 5 & 5 & 2 & 3 & 3 & 2 \\
\hline \multicolumn{12}{|c|}{ Medidas de composición } \\
\hline$\% \mathrm{EPT}$ & 73 & 90 & 95 & 98 & 95 & 68 & 43 & 69 & 99 & 96 & 98 \\
\hline \% Ephemeroptera & 68 & 86 & 84 & 87 & 92 & 47 & 32 & 68 & 96 & 87 & 94 \\
\hline$\%$ Trichoptera & 5 & 0 & 0 & 0 & 0 & 21 & 10 & 1 & 1 & 5 & 3 \\
\hline$\%$ Diptera & 23 & 10 & 0 & 2 & 0 & 31 & 54 & 29 & 1 & 3 & 1 \\
\hline$\%$ Chironomidae & 23 & 6 & 0 & 0 & 0 & 26 & 50 & 26 & 1 & 1 & 1 \\
\hline \multicolumn{12}{|c|}{ Medidas de tolerancia } \\
\hline BMWP & 17.9 & 30.4 & 26.7 & 26.7 & 33.1 & 39.2 & 46.9 & 24.3 & 37.0 & 43.4 & 50.1 \\
\hline BMWP-ASPT & 6.0 & 7.6 & 8.9 & 8.9 & 6.6 & 4.4 & 5.2 & 6.1 & 6.2 & 6.2 & 7.2 \\
\hline IBMWP & 16 & 36 & 24 & 38 & 37 & 58 & 69 & 39 & 46 & 51 & 52 \\
\hline IBMWP-ASPT & 5.3 & 7.2 & 8 & 7.6 & 7.4 & 5.3 & 5.8 & 6.5 & 6.6 & 6.4 & 6.5 \\
\hline $\mathrm{BMWP} / \mathrm{CR}$ & 25 & 40 & 28 & 42 & 40 & 71 & 92 & 43 & 65 & 60 & 78 \\
\hline BMWP/CR-ASPT & 5 & 6.7 & 7 & 7 & 6.7 & 5.5 & 5.8 & 6.1 & 6.5 & 6 & 6.5 \\
\hline $\mathrm{BMWP}^{\mathrm{THAI}}$ & 16 & 26 & 24 & 24 & 35 & 38 & 51 & 21 & 40 & 45 & 46 \\
\hline BMWP THAI-ASPT & 5.3 & 6.5 & 8.0 & 8.0 & 7.0 & 4.8 & 5.1 & 5.3 & 5.7 & 5.6 & 5.8 \\
\hline BMWP/Col & 33 & 45 & 33 & 47 & 49 & 75 & 101 & 48 & 75 & 72 & 90 \\
\hline BMWP/Col-ASPT & 6.6 & 7.5 & 8.3 & 7.8 & 8.2 & 6.8 & 6.7 & 6.9 & 7.5 & 7.2 & 7.5 \\
\hline NEPBIOS & 25 & 25 & 24 & 24 & 32 & 66 & 78 & 23 & 53 & 53 & 60 \\
\hline NEPBIOS-ASPT & 6.3 & 6.3 & 8.0 & 8.0 & 8.0 & 6.6 & 7.1 & 5.8 & 6.6 & 6.6 & 6.7 \\
\hline SASS5 & 32 & 59 & 42 & 63 & 56 & 86 & 107 & 55 & 84 & 92 & 93 \\
\hline SASS-ASPT & 8.0 & 9.8 & 10.5 & 10.5 & 9.3 & 7.8 & 8.2 & 9.2 & 9.3 & 9.2 & 9.3 \\
\hline SIGNAL2 score (abundancia) & 3.8 & 4.2 & 4.2 & 5.2 & 4.5 & 5.3 & 5.9 & 5.4 & 5.7 & 5.8 & 5.4 \\
\hline SIGNAL2 score (no abundancia) & 6.0 & 7.2 & 7.7 & 7.6 & 7.0 & 5.5 & 6.3 & 7.0 & 6.9 & 6.8 & 6.8 \\
\hline
\end{tabular}

(c) (P)




\section{Revista de Desde
since 1980 CIENCIAS AMBIENTALES Tropical Journal of Environmental Sciences}

Revista de Ciencias Ambientales (Trop J Environ Sci). EISSN: 2215-3896. (Enero-Junio, 2019) . Vol 53(1): 1-22. D0l: http://dx.doi.org/10.15359/rca.53-1.1 Open Access: www.revistas.una.ac.cr/ambientales EMAlL: revista.ambientales@una.ac.c Lafuente W., Soto L. M., López C., Domínguez-Granda L.

Apéndice 2. Índices bióticos calculados para cada estación de muestreo para el año 2012

\begin{tabular}{|c|c|c|c|c|c|c|c|c|c|c|c|}
\hline Código de estación & E1 & E2 & E3 & $\mathrm{E} 4$ & E5 & E6 & E7 & E8 & E9 & E10 & E11 \\
\hline \multicolumn{12}{|c|}{ Medidas de abundancia } \\
\hline N. ${ }^{\circ}$ de individuos & 22 & 50 & 19 & 94 & 39 & 257 & 393 & 185 & 1098 & 386 & 740 \\
\hline \multicolumn{12}{|l|}{ Medidas de diversidad } \\
\hline Margalef & 2.1 & 1.4 & 1.8 & 1.8 & 1.9 & 3.4 & 3.0 & 2.2 & 1.5 & 2.0 & 2.3 \\
\hline Shannon-Wiener & 1.6 & 1.5 & 1.5 & 1.2 & 1.6 & 2.2 & 2.2 & 1.7 & 1.1 & 1.7 & 1.6 \\
\hline Simpson & 0.8 & 0.8 & 0.7 & 0.6 & 0.9 & 0.8 & 0.9 & 0.8 & 0.5 & 0.8 & 0.7 \\
\hline Evenness & 0.9 & 1.0 & 0.7 & 0.7 & 1.0 & 0.8 & 0.8 & 0.9 & 0.5 & 0.7 & 0.6 \\
\hline \multicolumn{12}{|c|}{ Medidas de riqueza de taxa } \\
\hline Número de taxa & 6 & 5 & 9 & 6 & 5 & 18 & 16 & 7 & 8 & 13 & 13 \\
\hline N. ${ }^{\circ}$ EPT taxa & 4 & 5 & 5 & 4 & 4 & 10 & 10 & 4 & 6 & 8 & 9 \\
\hline N. ${ }^{\circ}$ Ephemeroptera taxa & 3 & 3 & 3 & 3 & 3 & 3 & 3 & 2 & 3 & 3 & 3 \\
\hline N. ${ }^{\circ}$ Trichoptera taxa & 1 & 1 & 1 & 0 & 0 & 6 & 6 & 1 & 2 & 4 & 5 \\
\hline N. ${ }^{\circ}$ Diptera Taxa & 0 & 0 & 3 & 1 & 0 & 5 & 3 & 3 & 1 & 1 & 3 \\
\hline \multicolumn{12}{|c|}{ Medidas de composición } \\
\hline$\%$ EPT & 82 & 100 & 89 & 88 & 88 & 57 & 62 & 47 & 95 & 92 & 96 \\
\hline \% Ephemeroptera & 73 & 65 & 83 & 76 & 63 & 41 & 33 & 33 & 90 & 71 & 70 \\
\hline$\%$ Trichoptera & 9 & 12 & 1 & 0 & 0 & 15 & 25 & 7 & 4 & 9 & 23 \\
\hline$\%$ Diptera & 0 & 0 & 4 & 6 & 0 & 31 & 26 & 53 & 1 & 0 & 4 \\
\hline$\%$ Chironomidae & 0 & 0 & 2 & 6 & 0 & 1 & 5 & 7 & 0 & 0 & 2 \\
\hline \multicolumn{12}{|c|}{ Medidas de tolerancia } \\
\hline BMWP & 25.1 & 33.3 & 47.7 & 30.4 & 33.1 & 61.4 & 57.9 & 21.5 & 39.7 & 44.0 & 57.9 \\
\hline BMWP-ASPT & 8.4 & 8.3 & 6.8 & 7.6 & 8.3 & 6.1 & 6.4 & 5.4 & 7.9 & 7.3 & 6.4 \\
\hline IBMWP & 24 & 29 & 56 & 26 & 29 & 84 & 75 & 31 & 52 & 41 & 75 \\
\hline IBMWP-ASPT & 8 & 7.3 & 7 & 6.5 & 7.3 & 6 & 6.8 & 6.2 & 7.4 & 5.9 & 6.8 \\
\hline $\mathrm{BMWP} / \mathrm{CR}$ & 40 & 33 & 59 & 35 & 33 & 106 & 102 & 46 & 56 & 77 & 81 \\
\hline BMWP/CR-ASPT & 6.7 & 6.6 & 6.6 & 5.8 & 6.6 & 5.9 & 6.4 & 6.6 & 7.0 & 5.9 & 6.2 \\
\hline BMWP & 29 & 29 & 46 & 26 & 29 & 62 & 61 & 21 & 34 & 43 & 57 \\
\hline BMWP ${ }^{\text {THAI}}-A S P T$ & 7.3 & 7.3 & 6.6 & 6.5 & 7.3 & 5.6 & 6.1 & 5.3 & 6.8 & 6.1 & 6.3 \\
\hline BMWP/Col & 48 & 40 & 69 & 40 & 39 & 120 & 112 & 48 & 63 & 95 & 97 \\
\hline BMWP/Col-ASPT & 8.0 & 8.0 & 7.7 & 6.7 & 7.8 & 7.1 & 7 & 6.9 & 7.9 & 7.3 & 7.5 \\
\hline NEPBIOS & 27 & 30 & 45 & 25 & 32 & 77 & 77 & 33 & 38 & 67 & 62 \\
\hline NEPBIOS-ASPT & 6.8 & 7.5 & 6.4 & 6.3 & 8 & 7.0 & 7.0 & 6.6 & 7.6 & 7.4 & 6.9 \\
\hline SASS5 & 52 & 54 & 72 & 56 & 50 & 111 & 116 & 55 & 77 & 83 & 96 \\
\hline SASS-ASPT & 10.4 & 10.8 & 9.0 & 9.3 & 10.0 & 7.9 & 8.9 & 9.2 & 11.0 & 9.2 & 8.7 \\
\hline SIGNAL2 score (abundancia) & 4.3 & 5.5 & 6.0 & 4.9 & 6.0 & 6.1 & 6.3 & 6.6 & 5.5 & 5.6 & 5.7 \\
\hline SIGNAL2 score (no abundancia) & 5.8 & 7.3 & 6.9 & 5.8 & 7.5 & 6.2 & 6.6 & 6.8 & 7.9 & 6.3 & 6.8 \\
\hline
\end{tabular}




\section{Revista de Since 1980 CIENCIAS AMBIENTALES Tropical Journal of Environmental Sciences}

Revista de Ciencias Ambientales (Trop J Environ Sci). EISSN: 2215-3896. (Enero-Junio, 2019) . Vol 53(1): 1-22. D0l: http://dx.doi.org/10.15359/rca.53-1.1 Open Access: www.revistas.una.ac.cr/ambientales EMAlL: revista.ambientales@una.ac.c Lafuente W., Soto L. M., López C., Domínguez-Granda L.

Apéndice 3. Valores de tolerancia de taxa para el año 2011

\begin{tabular}{|c|c|c|c|c|c|c|c|c|}
\hline \multicolumn{3}{|c|}{ Métodos de zonas templadas } & \multicolumn{6}{|c|}{ Métodos de zonas tropicales } \\
\hline & $\begin{array}{l}\text { BMWP } \\
\text { (revised) }\end{array}$ & IBMWP & $\begin{array}{c}\text { BMWP/ } \\
\text { COL }\end{array}$ & SASS5 & NEPBIOS & $\begin{array}{c}\text { BMWP/ } \\
\text { CR }\end{array}$ & $\begin{array}{c}\text { BMWP- } \\
\text { THAI }\end{array}$ & SIGNAL2 \\
\hline \multicolumn{9}{|l|}{ Coleoptera } \\
\hline Elmidae & 6,4 & 5 & 6 & 8 & 8 & 5 & 5 & 7 \\
\hline \multicolumn{9}{|l|}{ Diptera } \\
\hline Chironomidae & 3,7 & 2 & 2 & 2 & 1 & 2 & 2 & 3 \\
\hline Empididae & & 4 & 4 & 6 & & 4 & & 5 \\
\hline Tipulidae & & 5 & 3 & 5 & 7 & 4 & 5 & 5 \\
\hline Blepharoceridae & & 10 & 10 & 15 & & 10 & & 10 \\
\hline Simullidae & & 5 & 8 & 5 & 7 & 4 & 5 & 5 \\
\hline \multicolumn{9}{|l|}{ Ephemeroptera } \\
\hline Baetidae & 5,3 & 4 & 7 & 12 & 7 & 5 & 4 & 5 \\
\hline Leptohyphidae & & & 7 & 9 & & 5 & & \\
\hline Leptophlebiidae & 8,9 & 10 & 9 & 9 & 7 & 8 & 10 & 8 \\
\hline \multicolumn{9}{|l|}{ Lepidoptera } \\
\hline Pyralidae & & & 5 & 12 & & 5 & & 3 \\
\hline \multicolumn{9}{|l|}{ Odonata } \\
\hline Gomphidae & & 8 & 10 & 6 & & 7 & 6 & 5 \\
\hline \multicolumn{9}{|l|}{ Plecoptera } \\
\hline Perlidae & 12,5 & 10 & 10 & 12 & 10 & 10 & 10 & 10 \\
\hline \multicolumn{9}{|l|}{ Trichoptera } \\
\hline Hydropsychidae & 6,6 & 5 & 7 & 12 & 6 & 5 & 5 & 6 \\
\hline Hydroptilidae & 6,7 & 6 & 8 & 6 & 6 & 6 & 6 & 4 \\
\hline Hydrobiosidae & & & 9 & & 8 & 10 & & 8 \\
\hline Xiphocentronidae & & & 9 & & & 6 & & \\
\hline Helicopsychidae & & & 8 & & 10 & 5 & & 8 \\
\hline Glossosomatidae & & 8 & 7 & & & 8 & & 9 \\
\hline Amphipoda & 4,5 & 6 & & 13 & 7 & 7 & & 3 \\
\hline \multicolumn{9}{|l|}{ Arachnoidea } \\
\hline \multicolumn{9}{|l|}{ Megaloptera } \\
\hline Corydalidae & & & 6 & 8 & 7 & 6 & 4 & 7 \\
\hline Oligochaeta & 3,5 & 1 & & 1 & & 1 & 1 & 2 \\
\hline
\end{tabular}

(cc)




\section{Revista de CIENCIAS AMBIENTALES Tropical Journal of Environmental Sciences}

Revista de Ciencias Ambientales (Trop J Environ Sci). EISSN: 2215-3896. (Enero-Junio, 2019) . Vol 53(1): 1-22. D0l: http://dx.doi.org/10.15359/rca.53-1.1 Open Access: www.revistas.una.ac.cr/ambientales EMAlL: revista.ambientales@una.ac.c Lafuente W., Soto L. M., López C., Domínguez-Granda L.

Apéndice 4. Valores de tolerancia de taxa para el año 2012

\begin{tabular}{|c|c|c|c|c|c|c|c|c|}
\hline \multicolumn{3}{|c|}{ Métodos de zonas templadas } & \multicolumn{6}{|c|}{ Métodos de zonas tropicales } \\
\hline & $\begin{array}{l}\text { BMWP } \\
\text { (revised) }\end{array}$ & IBMWP & $\begin{array}{c}\text { BMWP/ } \\
\text { COL }\end{array}$ & SASS5 & NEPBIOS & $\begin{array}{c}\text { BMWP/ } \\
\text { CR }\end{array}$ & $\underset{\text { THAI }}{\text { BMWP- }}$ & SIGNAL2 \\
\hline \multicolumn{9}{|l|}{ Coleoptera } \\
\hline Elmidae & 6.4 & 5 & 6 & 8 & 8 & 5 & 5 & 7 \\
\hline Staphylinidae & & & 6 & & & 4 & & 3 \\
\hline Psephenidae & & & 10 & 10 & 8 & 7 & 5 & 6 \\
\hline \multicolumn{9}{|l|}{ Diptera } \\
\hline Chironomidae & 3.7 & 2 & 2 & 2 & 1 & 2 & 2 & 3 \\
\hline Dolichopodidae & & 4 & 4 & & & 4 & & 3 \\
\hline Empididae & & 4 & 4 & 6 & & 4 & & 5 \\
\hline Tipulidae & & 5 & 3 & 5 & 7 & 4 & 5 & 5 \\
\hline Blepharoceridae & & 10 & 10 & 15 & & 10 & & 10 \\
\hline Simullidae & & 5 & 8 & 5 & 7 & 4 & 5 & 5 \\
\hline \multicolumn{9}{|l|}{ Ephemeroptera } \\
\hline Baetidae & 5.3 & 4 & 7 & 12 & 7 & 5 & 4 & 5 \\
\hline Leptohyphidae & & & 7 & 9 & & 5 & & \\
\hline Leptophlebiidae & 8.9 & 10 & 9 & 9 & 7 & 8 & 10 & 8 \\
\hline \multicolumn{9}{|l|}{ Hemiptera } \\
\hline Naucoridae & 4.3 & 3 & 7 & 7 & 4 & 4 & 5 & 2 \\
\hline \multicolumn{9}{|l|}{ Lepidoptera } \\
\hline Pyralidae & & & 5 & 12 & & 5 & & 3 \\
\hline \multicolumn{9}{|l|}{ Plecoptera } \\
\hline Perlidae & 12.5 & 10 & 10 & 12 & 10 & 10 & 10 & 10 \\
\hline \multicolumn{9}{|l|}{ Trichoptera } \\
\hline Hydropsychidae & 6.6 & 5 & 7 & 12 & 6 & 5 & 5 & 6 \\
\hline Hydroptilidae & 6.7 & 6 & 8 & 6 & 6 & 6 & 6 & 4 \\
\hline Odontoceridae & 10.9 & 10 & 10 & & 5 & 10 & 10 & 7 \\
\hline Hydrobiosidae & & & 9 & & 8 & 10 & & 8 \\
\hline Xiphocentronidae & & & 9 & & & 6 & & \\
\hline Helicopsychidae & & & 8 & & 10 & 5 & & 8 \\
\hline Glossosomatidae & & 8 & 7 & & & 8 & & 9 \\
\hline Oligochaeta & 3.5 & 1 & & 1 & & 1 & 1 & 2 \\
\hline
\end{tabular}

(cc)

